\title{
Distance Measurements in Multiply Labeled Crystalline Cytidines by Dipolar Recoupling Solid State NMR
}

\author{
S. R. Kiihne, ${ }^{\dagger}$ K. B. Geahigan, ${ }^{\ddagger}$ N. A. Oyler, H. Zebroski, ${ }^{\S}$ M. A. Mehta," and G. P. Drobny* \\ Departments of Chemistry and Physics, University of Washington, Seattle, Washington 98195
}

Received: March 1, 1999

\begin{abstract}
To systematically explore the effects of spin system size and geometry on the precision and accuracy of two-dimensional solid state NMR distance measurements, we have applied two homonuclear dipolar recoupling experiments, 2D DRAWS and 2D RFDR, to five polycrystalline samples of uniformly or selectively ${ }^{13} \mathrm{C}$ labeled cytidine. Distance information has been obtained from the intensities and time behavior of crosspeaks observed in the resulting two-dimensional spectra. The experimental cross-peak buildup curves obtained from these crystalline cytidine samples have been analyzed by comparison with simulations. In uniformly ${ }^{13} \mathrm{C}$-labeled cytidine, indirect coherence transfer mechanisms lead to low-precision distance measurements not unlike those measured in solution state NOESY experiments. In the selectively labeled cytidines, the distance measurements are considerably more precise, allowing the possibility of very accurate structure determinations from selectively or randomly labeled spin systems. Of the two techniques, the 2D DRAWS method allows identification of indirect coherence transfer mechanisms that hinder accurate distance measurement.
\end{abstract}

\section{Background}

Solid state NMR homonuclear dipolar recoupling techniques are a means of studying the structures of molecules in amorphous solids, membrane or surface-bound biopolymers, or other systems that are not easily studied by solution NMR or X-ray crystallography. ${ }^{1-7}$ Multidimensional dipolar recoupling NMR techniques, developed by extension of existing onedimensional experiments into two or more dimensions, $, 3,6,8-10$ make possible the simultaneous measurement of many distances in uniformly isotopically labeled samples. Simulations have shown that multidimensional dipolar recoupling experiments may have sufficient resolution to allow the assignment of relatively large spin systems. ${ }^{8}$ However, although the precision and accuracy of solid state dipolar recoupling distance measurements of inhomogeneously broadened systems have been discussed in detail $1^{11-13}$ the accuracy of distance measurements in multidimensional dipolar recoupling experiments has not been explored in a systematic, quantitative fashion.

To determine the limitations of two-dimensional solid state dipolar recoupling experiments for quantifying internuclear distances, we have applied two different, dipolar recoupling pulse sequences to a series of uniformly and selectively ${ }^{13} \mathrm{C}$ labeled, cytidine monomers. These samples include doubly, triply, and uniformly ${ }^{13} \mathrm{C}$-labeled (i.e., $9{ }^{13} \mathrm{C}$ spins) cytidines, allowing an analysis of different quantitation methods and their sensitivity to indirect coherence transfer pathways and to spin system size. Two 3 -spin systems, cytidine- $2,1^{\prime}, 3^{\prime}-{ }^{13} C_{3}$ and

\footnotetext{
* To whom correspondence should be addressed.

† Present address: Chemistry Department, University of Virginia, Charlottesville, VA 22901.

† Present address: $3 \mathrm{M}$ Specialty Materials Division, $3 \mathrm{M}$ Chemicals, $3 \mathrm{M}$ Center, Building 236-2B-11 St. Paul, MN 55125-1000.

$\S$ Present address: Chiroscience Research and Development, 1631 220th St. SE, Bothell, WA 98021

" Present address: Department of Chemistry, Oberlin College, Oberlin, $\mathrm{OH} 44074$.
}

cytidine-2, $2^{\prime}, 5^{\prime}-{ }^{13} C_{3}$ (Figure 1), have been prepared to probe the effects of spin system geometry. To minimize the effects of line broadening resulting from structural heterogeneity, all experiments were carried out on crystalline samples. This also allows both a direct comparison between distances measured by solid state NMR and by X-ray crystallography, and a quantitation of the relative error of the NMR-derived distances.

To compare pulse sequence effects, cross-peak buildup curves are obtained with two dipolar recoupling methods: dipolar recoupling with a windowless sequence (DRAWS $)^{7}$ and radio frequency driven dipolar recoupling (RFDR). ${ }^{2}$ These two techniques have different coherence transfer mechanisms, which lead to significantly different results in two-dimensional experiments. In RFDR experiments dipolar recoupling occurs via a zero quantum Hamiltonian. As a result, indirect coherence transfer pathways are additive, and cross-peak intensities are equalized at long mixing times. In contrast, the DRAWS experiment recouples with an effective Hamiltonian containing both double and zero quantum terms and yields negative crosspeaks for direct coherence transfer paths. The cross-peak sign alternates with the number of spins involved in indirect transfer mechanisms, ${ }^{6}$ leading to a cancelation of cross-peak coherences at long mixing times.

For each ${ }^{13} \mathrm{C}$-labeled cytidine we have analyzed the experimental cross-peak buildup curves by exact simulation and by approximation methods in order to compare the accuracy and precision achievable with different data analysis methods. Determining distances by exact simulation, although feasible with small spin systems, is less straightforward in larger spin systems for two reasons. First, the large number of input parameters, which is required to fully define the spin system and which must be varied in successive simulations leads to a very large parameter space. Second, exact simulation of the 2D experiments for large spin systems requires prohibitive amounts of computer time. A number of approximation techniques are discussed and compared. 


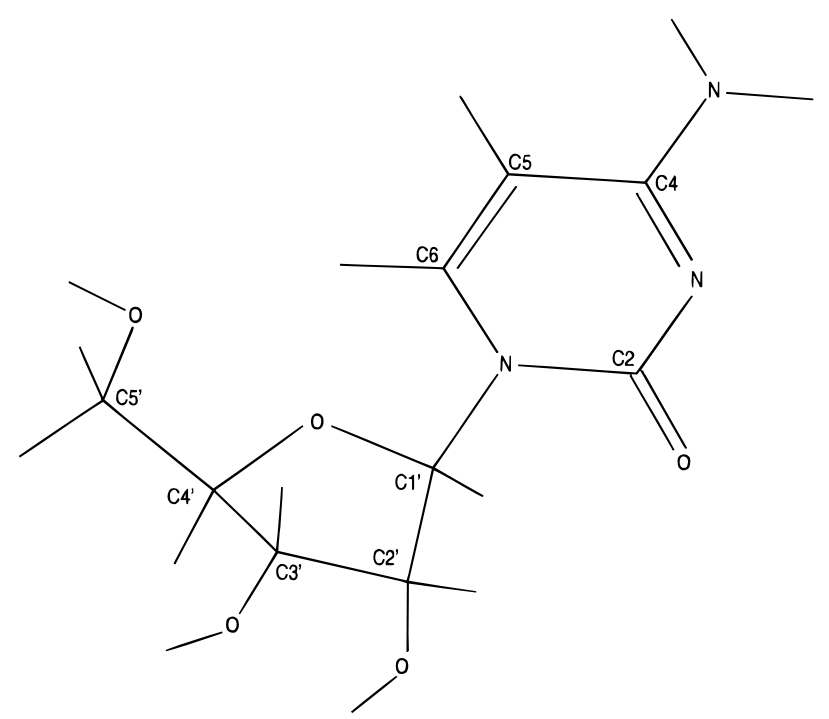

Figure 1. Cytidine crystal structure ${ }^{21}$ and atomic labeling scheme.

Combined, these approaches give a reasonably complete picture of the effects of the NMR pulse sequence, the spin system geometry and complexity, and the chemical shift and relaxation parameters on the distance information obtained from 2D dipolar recoupling experiments. These results can be used to generate optimal labeling strategies for further solid state NMR structural studies of more complex molecules and to determine the best methods for pursuing such studies.

\section{Materials and Methods}

Sample Synthesis. Selectively carbon-13-enriched cytidine nucleosides were prepared in five steps using methods compiled and modified by Kline and Serianni ${ }^{14}$ from previously published procedures. The syntheses of cytidine- $2,2^{\prime}-{ }^{13} C_{2}$, cytidine- $2, l^{\prime}$ ${ }^{13} C_{2}$, cytidine- $2,2^{\prime}, 5^{\prime}-{ }^{13} C_{3}$, and cytidine- $2,1^{\prime}, 3^{\prime}-{ }^{13} C_{3}$ were achieved using these methods with different combinations of ${ }^{13} \mathrm{C}$-labeled ribose and ${ }^{13} \mathrm{C}_{2}$-labeled cytosine. Cytosine- $2-{ }^{13} \mathrm{C}$, was purchased from Isotec, Inc. The selectively labeled sugars, D-ribose- $2^{\prime}, 5^{\prime}-$ ${ }^{13} C_{2}$, D-ribose- $1^{\prime}, 3^{\prime}-{ }^{13} C_{2}$, D-ribose- $2^{\prime}-{ }^{13} C$, and D-ribose- $-I^{\prime}-{ }^{13} C$ were purchased from Omecron, Inc. The ${ }^{13} \mathrm{C}$ enrichment level for cytosine-2- ${ }^{13} \mathrm{C}$ and for all labeled D-riboses was $99 \%$.

D-Ribose-2,5- ${ }^{13} C_{2}$, D-ribose-1,3- ${ }^{13} C_{2}$, D-ribose-2 $-{ }^{13} C$, and D-ribose- $1-{ }^{13} C$ were converted to mixtures of the methyl $\alpha$ - and $\beta$-D-ribofuranosides as described by Barker and Fletcher. ${ }^{15}$ The ${ }^{13} \mathrm{C}$-labeled methyl $\beta$-D-ribofuranoside anomer was converted to ${ }^{13} \mathrm{C}$-labeled 1 - $O$-acetyl $O$-benzoyl $\beta$-D-ribofuranoside (ABR) utilizing the techniques of Recondo and Rinderknecht. ${ }^{16} \mathrm{~N}^{4}$ protected acetylcytosine was prepared using the procedure of Codington and Fox. ${ }^{17}$ Silyation of the $\mathrm{N}^{4}$-protected acetylcytosine base with hexamethyldisilazane, condensation with ${ }^{13} \mathrm{C}$ labeled ABR, and removal of the acyl protecting groups with methanolic ammonia were accomplished using the methods of Vorbrüggen with some modifications to yield the desired $\beta$-cytidine anomer. ${ }^{18}$

Uniformly ${ }^{13} \mathrm{C}$ - and ${ }^{15} \mathrm{~N}$-labeled cytidine was synthesized using the procedure of Batey et al. ${ }^{19}$ with some alteration. Escherichia coli bacteria were grown on a minimal salt medium containing $\left({ }^{15} \mathrm{NH}_{4}\right)_{2} \mathrm{SO}_{4}(0.5 \mathrm{~g} / \mathrm{L})$ and $\left[{ }^{13} \mathrm{C}_{6}\right]$ glucose $(1.0 \mathrm{~g} / \mathrm{L})$, both obtained from Isotec. Wet-packed cells were lysed, and the nucleic acids were extracted with phenol, chloroform, and isoamyl alcohol. The procedure of Zimmer et al. ${ }^{20}$ was used to digest the nucleic acids into monophosphates. Digestion was monitored by reverse phase HPLC using a stepwise linear gradient of $10 \mathrm{mM}$ ammonium acetate and $60 \%$ acetonitrile/
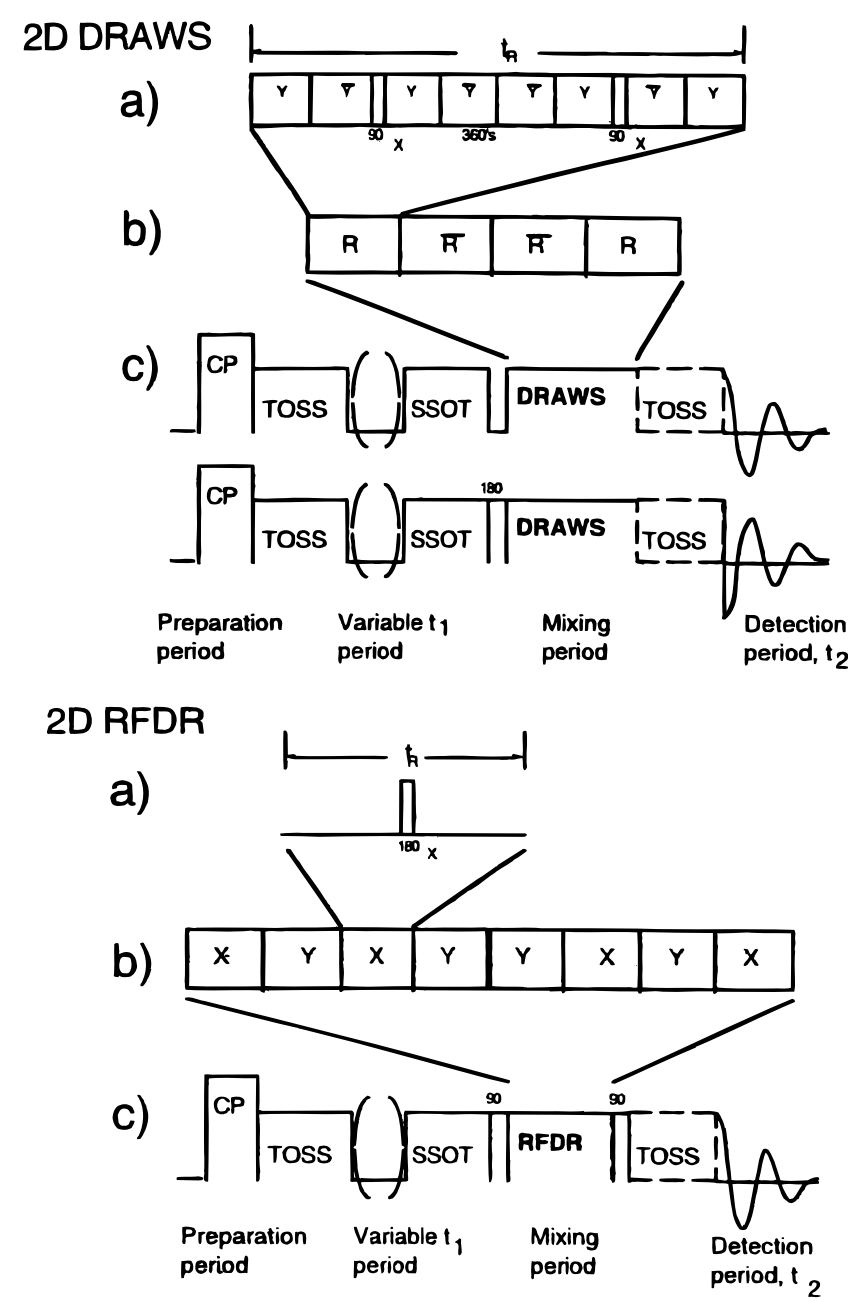

Figure 2. Carbon irradiation schemes for the 2D DRAWS and 2D RFDR pulse sequences. The basic dipolar recoupling pulse sequence is shown in (a). The appropriate supercycle is shown in (b), and the full $2 \mathrm{D}$ experiment is shown in (c). The final TOSS sequence (dotted lines) can be included to remove sidebands from the second dimension to improve resolution. The proton power levels and phase cycling are described in the text. The indicates a basic DRAWS sequence in which all phases are shifted by $180^{\circ}$.

$\mathrm{H}_{2} \mathrm{O}$. The deoxy- and ribonucleotides were separated by cisboronate affinity chromatography in which the ribonucleotides were eluted with $\mathrm{CO}_{2}$-acidified water. The isolated ribonucleotide monophosphates were dephosphorylated with calf intestinal phosphatase and separated by reverse phase HPLC using the same gradient described above. Residual HPLC salts were removed by repeated lyophylization. Isotopic enrichment (>95\%) and chemical purity were verified by ${ }^{1} \mathrm{H},{ }^{13} \mathrm{C}$, and ${ }^{15} \mathrm{~N}$ solution state NMR.

All cytidine samples were crystallized from $90 \%$ ethanol/ $10 \%$ water by slow evaporation. A crystal of cytidine- $2,2{ }^{\prime}-{ }^{13} C_{2}$ was analyzed by X-ray crystallography. The space group and unit cell dimensions were in agreement with those reported by Furberg et al. ${ }^{21}$

Spectroscopic Techniques and Data Processing. The NMR experiments were carried out on a home-built spectrometer operating at a ${ }^{13} \mathrm{C}$ Larmor frequency of $100.57 \mathrm{MHz}$, using the pulse sequences shown in Figure 2 . The ${ }^{13} \mathrm{C}$ radio frequency (RF) power level was ramped during cross-polarization to correct for long-term drift in the power amplifiers. A 2 ms crosspolarization period was used. The TOSS sequence of Raleigh et al. ${ }^{22}$ was used for sideband suppression in both dimensions of both 2D dipolar recoupling sequences. This has been found 
to minimize phase twisting effects and improve quantitation of cross-peaks. ${ }^{10}$ A rotor speed of $4.9 \mathrm{kHz}$ with a ${ }^{13} \mathrm{C}$ power level of $41.6 \mathrm{kHz}$ was used for all experiments, except during the cross-polarization period where the ${ }^{13} \mathrm{C}$ power was ramped through the Hartmann-Hahn condition. The ${ }^{1} \mathrm{H}$ power levels were adjusted to $50 \mathrm{kHz}$ during cross-polarization and were increased to $90-115 \mathrm{kHz}$ during the evolution, mixing, and detection periods. Purely absorptive peaks were obtained from 2D DRAWS experiments, as described previously. ${ }^{10}$ In 2D RFDR experiments, the $90^{\circ}$ pulses flanking the mixing period were phase cycled and the data processed by the method of States et al. ${ }^{23}$ A triply tuned ${ }^{1} \mathrm{H}-{ }^{15} \mathrm{~N}-\mathrm{X}$ probe from Doty Scientific with a $5 \mathrm{~mm}$ spinner assembly was used for all the experiments. Spin rates were controlled to within $\pm 5 \mathrm{~Hz}$ with a home-built spin rate controller. ${ }^{24}$ The ${ }^{1} \mathrm{H}$ pulses were provided by a Henry Radio 2004-A amplifier while the ${ }^{13} \mathrm{C}$ pulses were provided by a Kalmus LP-1000 amplifier.

In general, 512 transients were collected and the data were processed with Felix 2.10 software (Biosym Technologies). Each transient was multiplied with a shifted $\left(90^{\circ}\right)$ sine square apodization, and zero filled to a matrix size of 102451024 . Cross-peak footprints were determined at low contour levels and adjusted on each 2D data set to correct for spectrometer drift. Volumes were measured with the packaged routine.

Spin System Characterization and Simulation. Input parameters for the simulations were determined as follows: CSA tensor principal values were measured using the method of Herzfeld and Berger ${ }^{25}$ applied to a series of CP-MAS spectra of crystalline cytidine acquired at different sample spin rates. Internuclear distances were determined from the crystal structure of Furberg et al. ${ }^{21}$ The orientation of the cytidine C2 CSA tensor in the molecular frame was assumed to be analogous to that of the $\mathrm{C} 2$ tensor in 2 '-deoxythymidine. ${ }^{26}$ For the labeled sugar ring carbons, the CSA tensor orientations were assumed to be the same as those determined by McDowell et al. for the analogous resonances in dipotassium $\alpha$-D-glucose 1-phosphate dihydrate. ${ }^{27}$ The orientations of the dipolar and CSA tensors in the crystal frame were determined by rotation of a separate axis system relative to the crystal structure as displayed with InsightII software (Biosym Technologies).

Spin system simulations for 2 - and 3-spin systems also required two decay parameters, $R_{2 \mathrm{sq}}=1 / T_{2 \mathrm{sq}}$ and $R_{2 \mathrm{dq}}=1 / T_{2 \mathrm{dq}}$, which refer to the rates of decay of single quantum magnetization and double quantum coherence, respectively, resulting from a number of processes including relaxation, phase and amplitude errors in the dipolar recoupling pulse sequence, incomplete heteronuclear decoupling, etc.

For rare spins in small molecules $R_{2 \mathrm{sq}}$ may be measured by observing the rate of decay of the transverse magnetization of rare spins at natural abundance during the DRAWS pulse sequence. For rare spins in macromolecules, $R_{2 \text { sq }}$ may be more conveniently measured for individual spins by observing the rate of decay of the transverse magnetization of a single rare spin label during DRAWS irradiation, as described in Mehta et al. ${ }^{11}$ In doubly labeled molecules, $R_{2 \mathrm{sq}}$ may be also measured by selectively inverting one of the two transitions using a TOSS-SSOT pulse sequence ${ }^{30}$ and observing the decay of $\mathrm{I}_{x}-$ $\mathrm{S}_{x}$, as also described in Mehta et al. ${ }^{11}$

In least squares simulations of DRAWS data presented in this paper, both the internuclear distance $R(i, j)$ and $R_{2 \mathrm{dq}}$ are treated as adjustable parameters. This approach has been discussed by Heller et al. ${ }^{12}$ in the context of evaluating rotational resonance data obtained from inhomogeneously broadened systems. In this case an important consideration is the degree

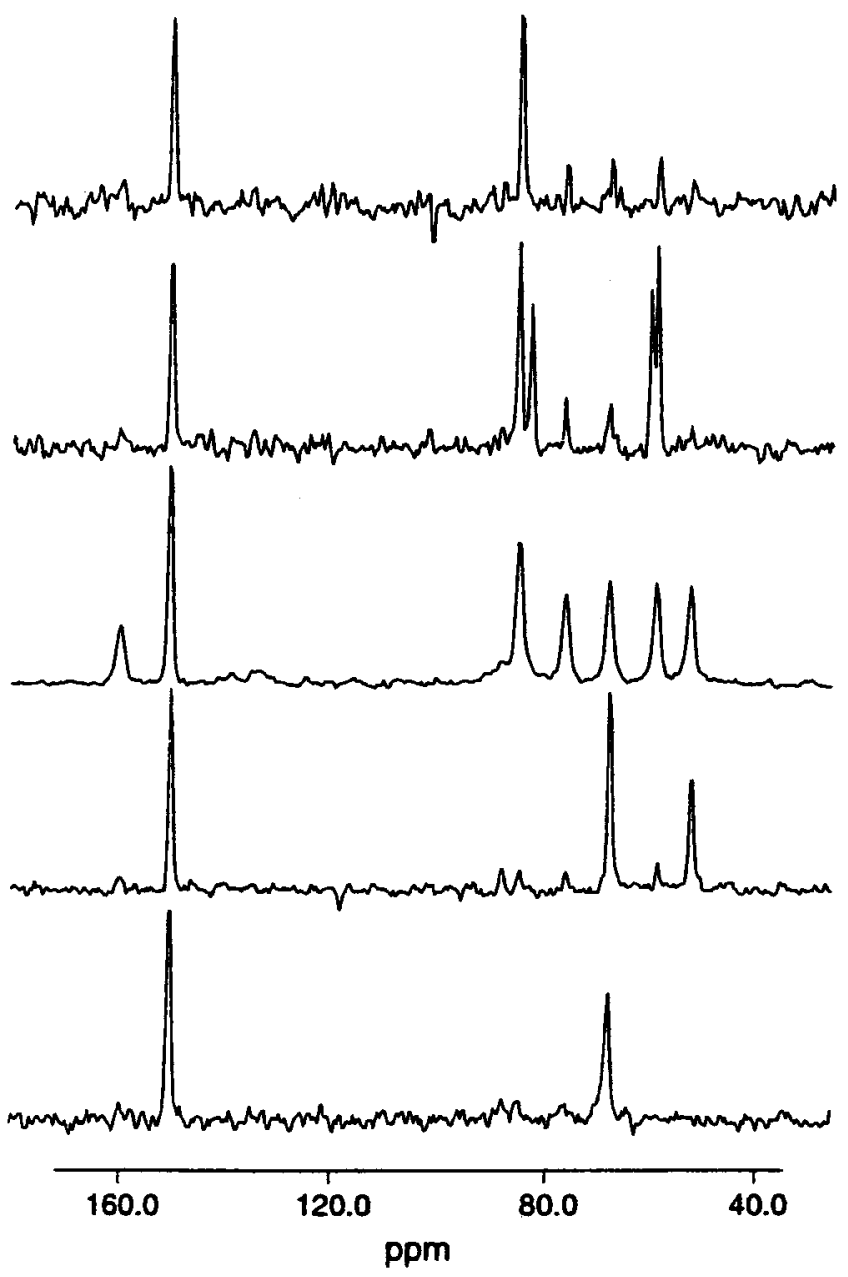

Figure 3. Solid state TOSS spectra of the isotopically labeled cytidine samples under the experimental conditions used in the 2D studies. From top to bottom, the samples are cytidine- $2,1^{\prime}-{ }^{13} C_{2}$, cytidine- $2,1^{\prime}, 3^{\prime}-{ }^{13} C_{3}$, cytidine- $U-{ }_{-1}^{13} C_{9},{ }^{15} N_{3}$, cytidine- $2,2^{\prime}, 5^{\prime}-{ }^{13} C_{3}$, and cytidine- $2,2^{\prime}-{ }^{13} C_{2}$. The signal-to-noise is typical of that used in the $2 \mathrm{D}$ experiments.

to which $R(i, j)$ and $R_{2 \mathrm{dq}}$ are correlated in least squares simulations of dipolar recoupling data, and the resulting degradation in the precision of the internuclear distance calculation when $R(i, j)$ and $R_{2 \mathrm{dq}}$ are strongly correlated. This topic has also been discussed by Heller et al. ${ }^{12}$ for correlations between $R(i, j)$ and $R_{2 \text { zq }}$ in rotational resonance studies of inhomogeneously broadened systems. In the following sections we will similarly quantify the uncertainty in the determination of the internuclear distance resulting from treating both $R(i, j)$ and $R_{2 \mathrm{dq}}$ as adjustable parameters in the least-squares fitting of DRAWS data for doubly labeled crystalline cytidines.

\section{Experimental Results}

CPMAS Data. Sideband suppressed spectra of the isotopically labeled cytidine compounds are shown in Figure 3. All labeled materials were diluted to $5-10 \%$ with natural abundance material in the solid state samples to eliminate intermolecular dipolar couplings. The peaks due to natural abundance material are clearly visible in some of the spectra. Both the $\mathrm{C}^{\prime}$ and $\mathrm{C}^{\prime}{ }^{\prime}$ peaks are split in all spectra of cytidine- $2,1^{\prime}, 3^{\prime}-{ }^{13} C_{3}$. These splittings are not observed in solution state ${ }^{13} \mathrm{C}$ spectra and are thus attributed to heterogeneity in the crystal. No splittings were observed in the spectra of cytidine- $2,1^{\prime}-{ }^{13} C_{2}$, which was synthesized and crystallized by the same methods. In spectra of the uniformly labeled sample, the C5 and C6 resonances have 
TABLE 1: Cytidine Chemical Shift and Relaxation Parameters $^{a}$

\begin{tabular}{lcrrrrrr}
\hline & $\sigma_{\text {iso }}$ & \multicolumn{1}{c}{$\sigma_{11}$} & \multicolumn{1}{c}{$\sigma_{22}$} & $\sigma_{33}$ & \multicolumn{1}{c}{$\alpha$} & \multicolumn{1}{c}{$\beta$} & $\gamma$ \\
\hline $\mathrm{C} 2$ & 154.3 & 214.0 & 155.8 & 93.1 & $180^{\circ}$ & $46^{\circ}$ & $123^{\circ}$ \\
$\mathrm{C}^{\prime}$ & 88.8 & 111.3 & 89.3 & 65.8 & $56^{\circ}$ & $73^{\circ}$ & $224^{\circ}$ \\
$\mathrm{C}^{\prime}$ & 71.9 & 55.2 & 70.6 & 89.9 & $322^{\circ}$ & $115^{\circ}$ & $195^{\circ}$ \\
$\mathrm{C}^{\prime}$ & 62.83 & 31.8 & 58.8 & 97.9 & $59^{\circ}$ & $169^{\circ}$ & $100^{\circ}$ \\
$\mathrm{C}^{\prime}$ & 56.3 & 26.7 & 51.7 & 90.5 & $37^{\circ}$ & $133^{\circ}$ & $347^{\circ}$
\end{tabular}

${ }^{a}$ Chemical shift parameters are given in ppm. The angles $\alpha, \beta$, and $\gamma$ orient the chemical shift tensor in the crystal frame.

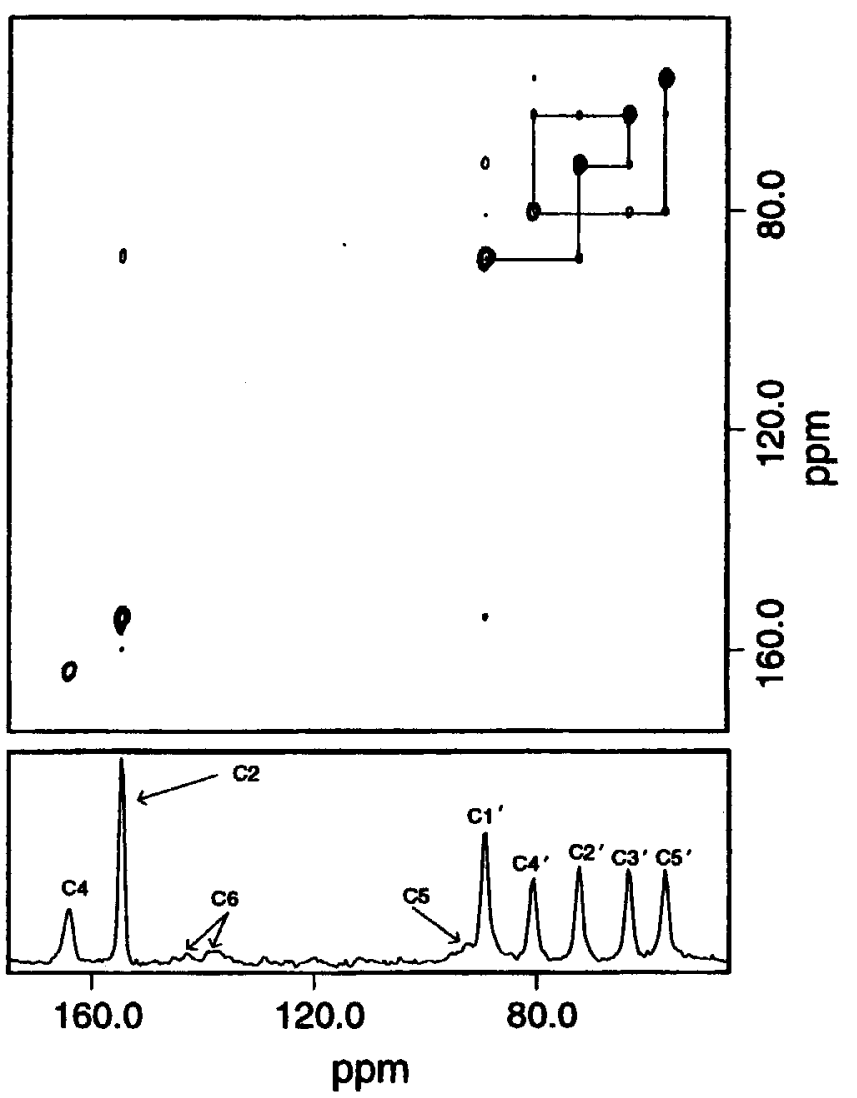

Figure 4. 2D RFDR spectrum of cytidine- $U-{ }^{13} C,{ }^{15} N$. The sugar ring assignments are based on the data, as shown by the connectivities superimposed on the 2D matrix.

relatively low signal-to-noise, and the C6 resonance is split. To avoid errors, neither the C5 nor C6 resonances were included in the distance measurements discussed below. CP-MAS spectra of the selectively labeled samples were collected at a series of spin rates, and the principle values of the CSA tensors were determined by the method of Herzfeld and Berger. ${ }^{25}$ The results are given in Table 1 .

Uniformly Labeled, 9-Spin System Spectral Assignment. The 2D RFDR spectrum of cytidine- $U-{ }^{13} C,{ }^{15} N$ after 2 cycles of RFDR (mixing period $=3.254 \mathrm{~ms}$ ) is shown in Figure 4 along with the chemical shift assignment of the sugar ring resonances. Correlations between directly coupled carbon atoms lead to the strong positive cross-peaks observed in the spectrum. In 2D DRAWS spectra, direct spin-spin correlations appear as strong, negative cross-peaks. Weaker correlations between more distant carbons appear as additional peaks. In the 2D RFDR spectrum, these peaks are all positive, so the assignment must be made on the basis of their relative intensities. In the 2D DRAWS spectra, the cross-peak sign depends on the primary magnetization transfer pathway. This allows the assignment of nearest neighbor sites based on the sign of the cross-peak. ${ }^{6}$

In cytidine, the carbon skeleton for the furanose moiety can
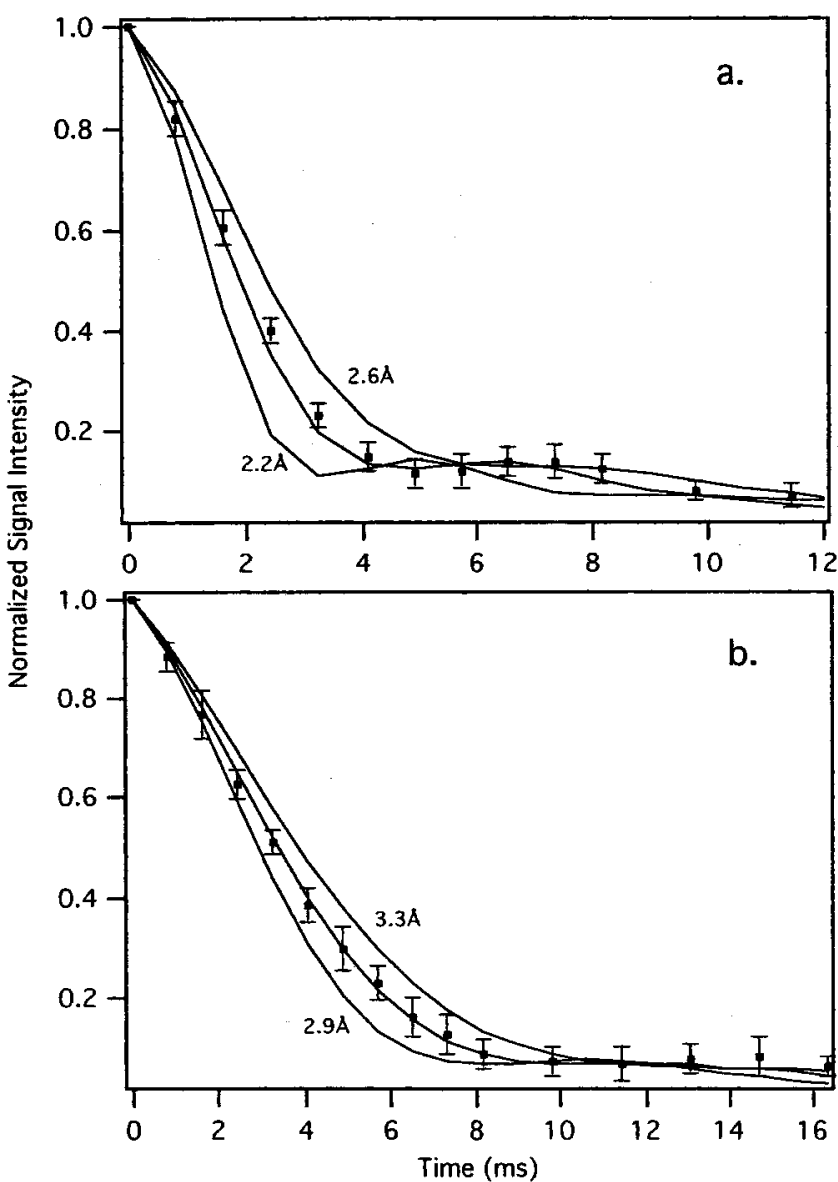

Figure 5. One-dimensional DRAWS distance measurements of the two doubly isotopically labeled cytidine samples. The cytidine- $2,1^{\prime}$ ${ }^{13} C_{2}$ DRAWS dephasing curve is shown in (a) and the cytidine-2, $2^{\prime}-$ ${ }^{13} C_{2}$ DRAWS dephasing curve is shown in (b).

be readily traced by inspection of the data set in Figure 4: $\mathrm{C} 1^{\prime}$ (88.8 ppm) is correlated with $\mathrm{C}^{\prime}$ (71.9 ppm), C2' with C3' (62.8

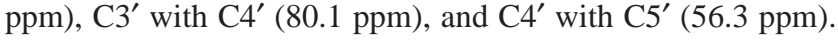
The relatively narrow and unsplit cross-peaks in the spectrum indicate the presence of only one molecular conformation in the crystal asymmetric unit, in accordance with X-ray data. ${ }^{21}$ In nucleic acids, the isotropic chemical shifts of the sugar ring resonances are indicative of the ring conformation, ${ }^{29}$ and the relatively upfield shifts of the $\mathrm{C}^{\prime}$ and $\mathrm{C}^{\prime}$ resonances in the cytidine spectra indicate that the sugar ring is in the C3' endo conformation characteristic of A-form nucleic acid structures. This also agrees with the ring conformation observed in the crystal structure. ${ }^{21}$

The C2 carbon (138.9 ppm) may be indentified by a strong cross-peak to $\mathrm{Cl}^{\prime}$ (88.8 ppm). Additionally, a very weak positive connectivity between the aromatic $\mathrm{C} 2$ nucleus and $\mathrm{C}^{\prime}$ on the ribose ring appears with two repetitions of DRAWS mixing. $\mathrm{C} 2$ has a strong cross-peak with a second aromatic carbon, which is certainly the $\mathrm{C} 4$ carbonyl $(164.0 \mathrm{ppm})$. The remaining unassigned downfield shifted auto-peak is assigned to C6 (138.9 ppm). The directly bonded C6 and C5 (92.0 ppm) atoms do not show a cross-peak in either RFDR or DRAWS data sets, probably as a result of low sensitivity and/or insufficient proton decoupling.

The initial assignment can be confirmed by inspection of the 2-cycle DRAWS spectrum. Additionally, positive cross-peaks due to indirect relay effects also appear in the 2-cycle DRAWS data set. The $\mathrm{C}^{\prime}-\mathrm{C} 3^{\prime}$ and $\mathrm{C}^{\prime}-\mathrm{C}^{\prime}$ sugar ring connectivities appear as strong positive cross-peaks, while the $\mathrm{C}^{\prime}-\mathrm{C}^{\prime}$ 
a. Cytidine $2,1^{\prime} \chi^{2}$ contour map

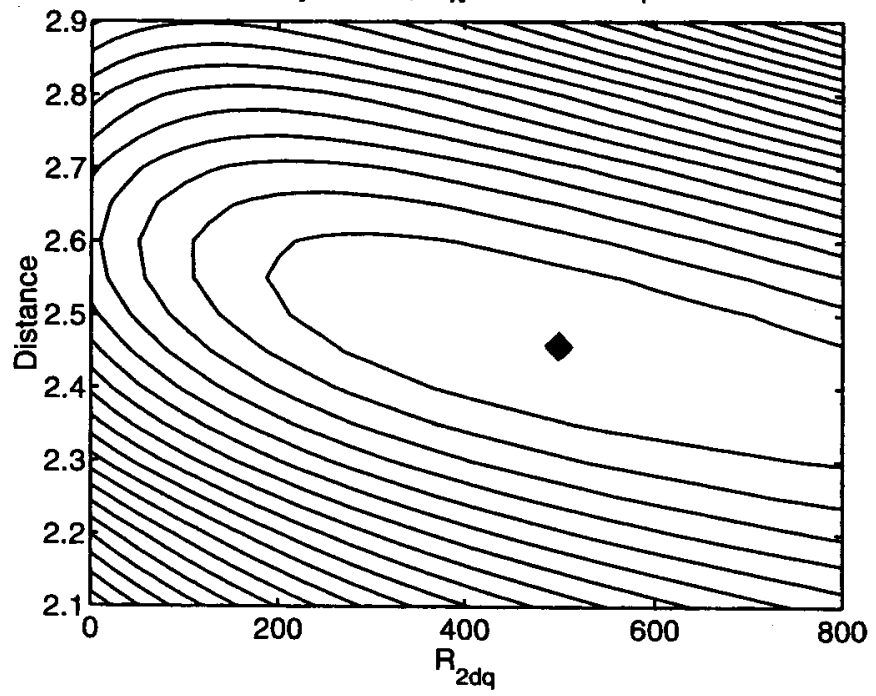

C. Cytidine $2,2^{\prime} \chi^{2}$ contour map

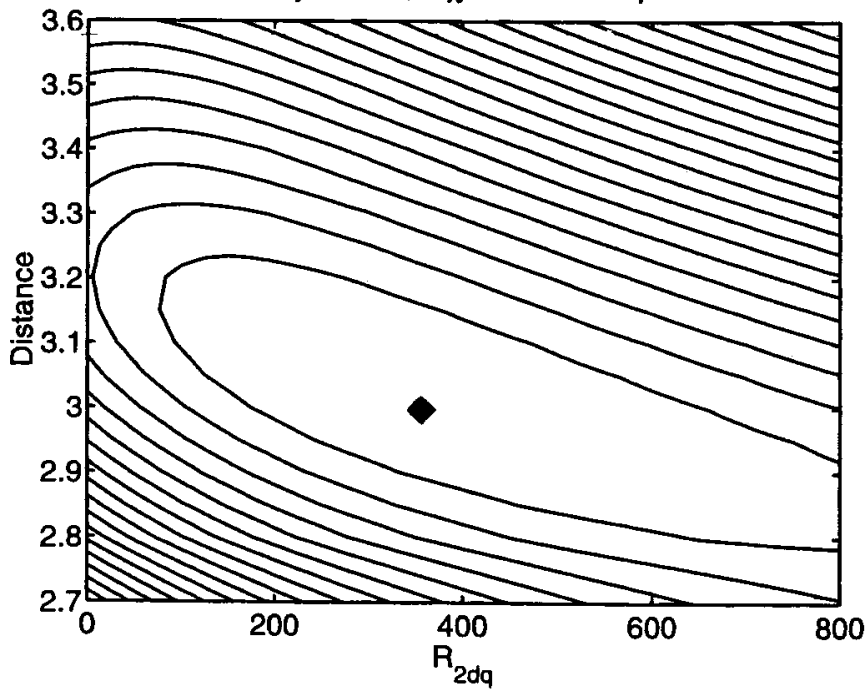

b. $\quad$ fits of the data for Cytidine $2,1^{\prime}$

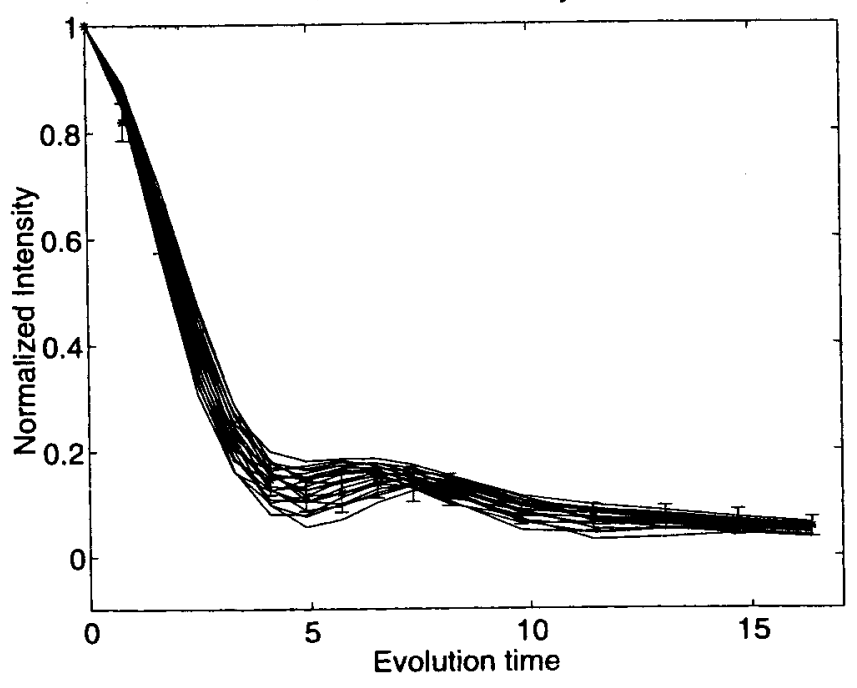

d. fits of the data for Cytidine $2,2^{\prime}$

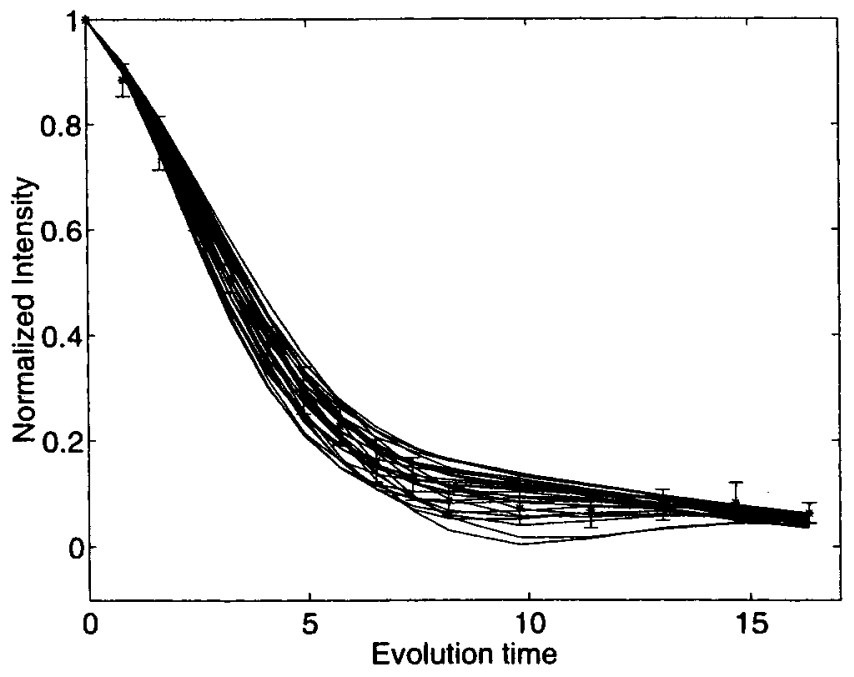

Figure 6. (a) $\chi^{2}$ contour map for the least-squares fitting of the DRAWS data for cytidine- $2,1^{\prime}-{ }^{13} C_{2}$ (see Figure 5a) where $r\left(2,1^{\prime}\right)$ and $R_{2 \mathrm{dq}}$ are treated as adjustable parameters. The $\chi^{2}$ minimum " $\chi^{2}(0)$ " is indicated by the filled diamond and was found to be 0.25 . The lowest contour indicated is at approximately $6 \chi^{2}(0)$, the second contour line is at approximately $12 \chi^{2}(0)$, etc. (b) The family of simulated DRAWS curves for $\chi^{2}$ values up to $6 \chi^{2}(0)$, superimposed on the experimental DRAWS data for cytidine- $2,1^{\prime}-{ }^{13} C_{2}$ (see Figure $5 \mathrm{a}$ ). Assuming 15 degrees of freedom (16 data points with the constraint that the first data point is unity), any fit for which $\chi^{2}>1.5$ has a probability of $<5 \%$ of being significant (see Figure C.4 in ref 28 or Table D in ref 34). (c) $\chi^{2}$ contour map for the least-squares fitting of DRAWS data for cytidine-2, $2^{\prime}-{ }^{13} C_{2}($ see Figure $5 \mathrm{~b}$ ) where $r\left(2,2^{\prime}\right)$ and $R_{2 \mathrm{dq}}$ are treated as adjustable parameters. The $\chi^{2}$ minimum $\chi^{2}(0)$ equals 0.2 and is indicated by the filled diamond. (d) The family of simulated DRAWS curves for $\chi^{2}$ values up to $8 \chi^{2}(0)=1.6$, superimposed on the experimental DRAWS data for cytidine-2,2'-13 $C_{2}$ (see Figure $5 b)$. Given 15 degrees of freedom, any fit for which $\chi^{2}>1.6$ has a probability of $<5-6 \%$ of being significant.

correlation is slightly weaker. Two weak negative connectivities between $\mathrm{C} 1^{\prime}-\mathrm{C}^{\prime}$ and $\mathrm{C} 2^{\prime}-\mathrm{C}^{\prime}$ are evidence of longer range couplings. A very faint negative correlation between $\mathrm{C} 2$ and $\mathrm{C}^{\prime}$ is also present in the 2-cycle DRAWS data set.

To measure exact internuclear distances, two-dimensional RFDR and DRAWS spectra of cytidine- $U-{ }^{13} C,{ }^{15} \mathrm{~N}$ were acquired at a series of mixing times corresponding to one, two, three, four, and five cycles of each dipolar recoupling pulse sequence. For each experiment, a matrix of 5125256 points was collected with a repetition time of $7 \mathrm{~s}$. Each $t_{1}$ time point was signal averaged over 16 scans. As in Figure 3, all peaks are well resolved and fully absorptive and there are few artifacts. Crosspeaks were observed for 97 of the 100 resonances.

Two-Spin Systems: One-Dimensional DRAWS Experimental Results and Simulations. The two doubly labeled cytidine samples, cytidine- $2,1^{\prime}-{ }^{13} C_{2}$ and cytidine- $2,2{ }^{\prime}-{ }^{13} C_{2}$ were studied by both one-, and two-dimensional dipolar recoupling methods. One-dimensional DRAWS dephasing curves and simulations for cytidine- $2,1^{\prime}-{ }^{13} C_{2}$ and cytidine- $2,2{ }^{\prime}-{ }^{13} C_{2}$ are shown in Figure 5a,b, respectively. To determine $R_{2 \mathrm{sq}}$ a control experiment was performed on each sample by inverting the $\mathrm{C} 2$ resonance with the TOSS-SSOT method ${ }^{30}$ before application of the DRAWS recoupling pulses. From the control experiment $R_{2 \mathrm{sq}}$ was determined to be approximately $100 \mathrm{~Hz}$ for C2.

A least-squares fitting of the DRAWS dephasing data for cytidine- $2, l^{\prime}-{ }^{13} C_{2}$, which treated both the internuclear distance $r(i, j)$ and $R_{2 \mathrm{dq}}$ as adjustable parameters, resulted in a $\mathrm{C} 2-\mathrm{C} 1^{\prime}$ distance $r\left(2,1^{\prime}\right)=2.46 \AA$ and $R_{2 \mathrm{dq}}=475 \mathrm{~Hz}$. The $\mathrm{C} 2-\mathrm{C}^{\prime}{ }^{\prime}$ distance determined from the crystal structure data is also 2.46 $\AA$. Figure $6 \mathrm{a}$ is a contour plot of $\chi^{2}$ for the least-squares fitting of DRAWS decay data of cytidine- $2,1^{\prime}-{ }^{13} C_{2}$ as a function of $r\left(2,1^{\prime}\right)$ and $R_{2 \mathrm{dq}}$, where $\chi^{2}$ is defined as the sum of the squares 
of the deviations of the simulated DRAWS decay data from the experimental data, i.e., $\chi^{2}=(1 / d) \sum_{i=1}^{m}\left[\left(O_{i}-E_{i}\right) / \sigma_{i}\right]^{2}$. In the $\chi^{2}$ equation $O_{i}$ is the value of the $i$ th experimental point in the DRAWS curve, $E_{i}$ is the simulated value for the $i$ th point in the DRAWS curve, $d$ is the number of degrees of freedom in the data set, and $s_{i}$ is the standard deviation of the distribution of measurements of the $i$ th point of the DRAWS curve. Other simulation parameters are given in Table 1.

Figure 6a shows that $\chi^{2}$ contours follow elliptical paths near the $\chi^{2}$ minimum, $\chi^{2}(0)$. If $r(i, j)$ and $R_{2 \mathrm{dq}}$ are not correlated and if the $\chi^{2}$ surface is parabolic near the minimum, the uncertainty in $r(i, j), \sigma_{r}^{2}$, may be determined from the relationship $1 / \sigma_{r}^{2}=$ $(1 / 2)\left(\mathrm{G} 2 \partial^{2} \chi^{2} / \partial r^{2}\right)$, where the curvature $\partial^{2} \chi^{2} / \partial r^{2}$ is evaluated near the $\chi^{2}$ minimum, ${ }^{28}$ indicated by the filled diamond in Figure 6a. Assuming $r(i, j)$ and $R_{2 \mathrm{dq}}$ are not correlated and using the value of the curvature $\partial^{2} \chi^{2} / \partial r^{2}$ at the $\chi^{2}$ minimum, we find $\sigma_{r}$ to be about $\pm 0.08 \AA$.

The degree of correlation between $r(i, j)$ and $R_{2 \mathrm{dq}}$ is indicated by the tilting of the semi-axes of the $\chi^{2}$ contour ellipses away from the coordinate axes. ${ }^{28}$ Figure 6 a shows that there is clearly some correlation between $r\left(2,1^{\prime}\right)$ and $R_{2 \mathrm{dq}}$. To estimate the degree to which the correlation between $R_{2 \mathrm{dq}}$ and $r\left(2,1^{\prime}\right)$ influences the uncertainty of $r\left(2,1^{\prime}\right)$, theoretical DRAWS decay curves are plotted in Figure $6 b$ for $\chi^{2}$ values up to $6 \chi^{2}(0)$. The cutoff value of $\chi^{2}=6 \chi^{2}(0)$ ensures that any simulation with a larger $\chi^{2}$ has a probability of less than $5 \%$ of being significant. Figure $6 \mathrm{~b}$ shows that DRAWS simulations with equal $\chi^{2}$, but differing $R_{2 \mathrm{dq}}$ show considerable variation in the initial slope of the dephasing curve, a region that is sensitive to internuclear distance. In comparing Figures $5 \mathrm{a}$ and $6 \mathrm{~b}$, it is clear that $r\left(2,1^{\prime}\right)$ in cytidine- $2,1^{\prime}-{ }^{13} C_{2}$ cannot be determined to better than about $\pm 0.15 \AA$ if both $r\left(2,1^{\prime}\right)$ and $R_{2 \mathrm{dq}}$ are treated as adjustable parameters.

A similar least-squares fitting of the DRAWS dephasing data for cytidine-2,2'-13 $C_{2}$ in which both $r\left(2,2^{\prime}\right)$ and $R_{2 \mathrm{dq}}$ were again treated as adjustable parameters, resulted in a $\mathrm{C} 2-\mathrm{C}^{\prime}$ distance of $3.03 \AA$, which is close to the distance of $3.18 \AA$ obtained from the crystal structure. ${ }^{21}$ The $R_{2 \mathrm{dq}}$ at the $\chi^{2}$ minimum, indicated by the filled diamond in Figure $6 \mathrm{c}$, was $375 \mathrm{~Hz}$. Again, assuming no correlation between $r\left(2,2^{\prime}\right)$ and $R_{2 \mathrm{dq}}$ the uncertainty in $\mathrm{R}, \sigma_{r}$, was obtained as before, by evaluating the curvature $\partial^{2} \chi^{2} / \partial r^{2}$ at the $\chi^{2}$ minimum. By this method the uncertainty was found to be $\sigma_{r}= \pm 0.14 \AA$.

However, the $\chi^{2}$ contour map for the least-squares fitting of the cytidine-2, $2^{\prime}-{ }^{13} C_{2}$ DRAWS data (Figure 6c) shows a somewhat stronger correlation between $r(i, j)$ and $R_{2 \mathrm{dq}}$ for cytidine-2, $2{ }^{\prime}-{ }^{13} C_{2}$ than for cytidine- $2,1^{\prime}-{ }^{13} C_{2}$, so the effect that this correlation has on the uncertainty of $R\left(2,2^{\prime}\right)$ must also be considered. Figure $6 \mathrm{~d}$ shows a superposition of DRAWS simulations for $\chi^{2}$ values up to $8 \chi^{2}(0)$, where this cutoff ensures that any simulation with a larger $\chi^{2}$ has a probability of less than $5-6 \%$ of being significant. A comparison of Figures $6 \mathrm{~d}$ and $5 \mathrm{~b}$ shows that if $R_{2 \mathrm{dq}}$ is freely adjusted together with $r\left(2,2^{\prime}\right)$, the uncertainty in the internuclear distance has increased to greater than $\pm 0.2 \AA$.

2D DRAWS Results for the 3-Spin System. To assess the influence of indirect coherence transfers on distance measurements by multidimensional solid state NMR, the two triply labeled cytidine samples were studied with both 2D DRAWS and 2D RFDR and the results were compared to measurements made on doubly labeled cytidine samples. As with the uniformly labeled sample, matrices of 5125256 points were collected with a repetition time of $7 \mathrm{~s}$. As in the doubly labeled samples, the mixing times were varied to account for the slower cross-peak buildup rates observed in these selectively labeled systems. For the cytidine- $2,1^{\prime}, 3^{\prime}-{ }^{13} C_{3}$ sample, mixing times were $1,2,4,6$, and 8 cycles of dipolar recoupling, while for cytidine- $2,2^{\prime}, 5^{\prime}$ ${ }^{13} C_{3}$ mixing times of $2,6,10,12$, and 16 cycles were used. Stack plots of simulated and experimental 2D DRAWS spectra obtained from cytidine- $2,1^{\prime}, 3^{\prime}-{ }^{13} C_{3}$ are shown in Figure $7 a, b$, respectively. The positive peaks in the spectra are shown on the left side of each figure, while the negative peaks are shown on the right. The relative peak intensity is the same throughout. The negative cross-peaks between the $\mathrm{C} 2$ and $\mathrm{C}^{\prime}{ }^{\prime}$ resonances and between the $\mathrm{C}^{\prime}$ and $\mathrm{C}^{\prime}$ ' resonances are clearly seen on the right. The relative sign of these peaks indicates direct transfer of coherence. A small, positive cross-peak between the $\mathrm{C} 2$ and C3' resonances is observed on the left side of the figure. This cross-peak corresponds to a relatively long distance (4.2 $\AA$ ), and the sign change indicates that its formation is dominated by an indirect coherence transfer mechanism. ${ }^{20}$

\section{Simulation and Analysis of Two-Dimensional NMR Data}

Theoretical Principles. We used several simulation techniques to analyze the cross-peak buildup curves obtained from the two-dimensional DRAWS and RFDR experiments. The simulation methods vary significantly in computation time requirements, and in the degree of accuracy in reproducing the data. As in the 1D simulations DRAWS shown in Figure 5, above, exact, numerical simulations of the density operator in a two-dimensional experiment entail propagation of the density matrix by a numerically integrated effective Hamiltonian over all the time periods of the $2 \mathrm{D}$ experiment.

$$
\rho(\tau)=U_{t_{2}} U_{\text {mix }} U_{t_{1}} \rho(0) U_{t_{1}}^{\dagger} U_{\text {mix }}^{\dagger} U_{t_{2}}^{\dagger}
$$

In each case a numerically exact propagator $U$ is obtained by multiplication of propagators over small time increments in a time-ordered fashion:

$$
\begin{aligned}
& U(\tau)=T \mathrm{e}^{-i \int_{0}^{r} \mathrm{~d} t^{\prime} H\left(t^{\prime}\right)}= \\
& \mathrm{e}^{-i H\left(t_{N}\right) \delta t_{N}} \ldots \mathrm{e}^{-i H\left(t_{2}\right) \delta t_{2}} \mathrm{e}^{-i H\left(t_{1}\right) \delta t_{1}}=\mathrm{e}^{-i H_{\text {eff }} \tau}
\end{aligned}
$$

where the effective Hamiltonian $H_{\text {eff }}$ is calculated by taking the matrix logarithm of the numerically calculated propagator, i.e., $H_{\text {eff }}=i \log (U) / \tau$. In such simulations, magic angle spinning is included as a time dependent reorientation of the spin system tensor interactions, typically requiring 60-70 integration increments for each rotor period. The propagator is calculated for each crystallite, for each time increment within a rotor period, and recycled to the extent possible over the full 2D simulation. Both the initial density matrix and the measured observable are sums of components due to all the spins in the system.

$$
\rho(0)=\sum_{i} I_{y}^{(i)}
$$

Such simulations yield full 2D data matrices that are processed in exactly the same way as experimental data. Such a simulation of a 2D DRAWS spectrum of cytidine- $2,1^{\prime}, 3^{\prime}-{ }^{13} C_{3}$ is shown in Figure 7. We will refer to this as a full matrix simulation.

A second simulation technique, which is referred to as a crosspeak buildup curve simulation, can save computational time significantly without simplifying the spin system Hamiltonian. An initial spin state consisting of a single spin is considered.

$$
\hat{O}=\rho_{i}(0)=I_{y}^{(i)}
$$



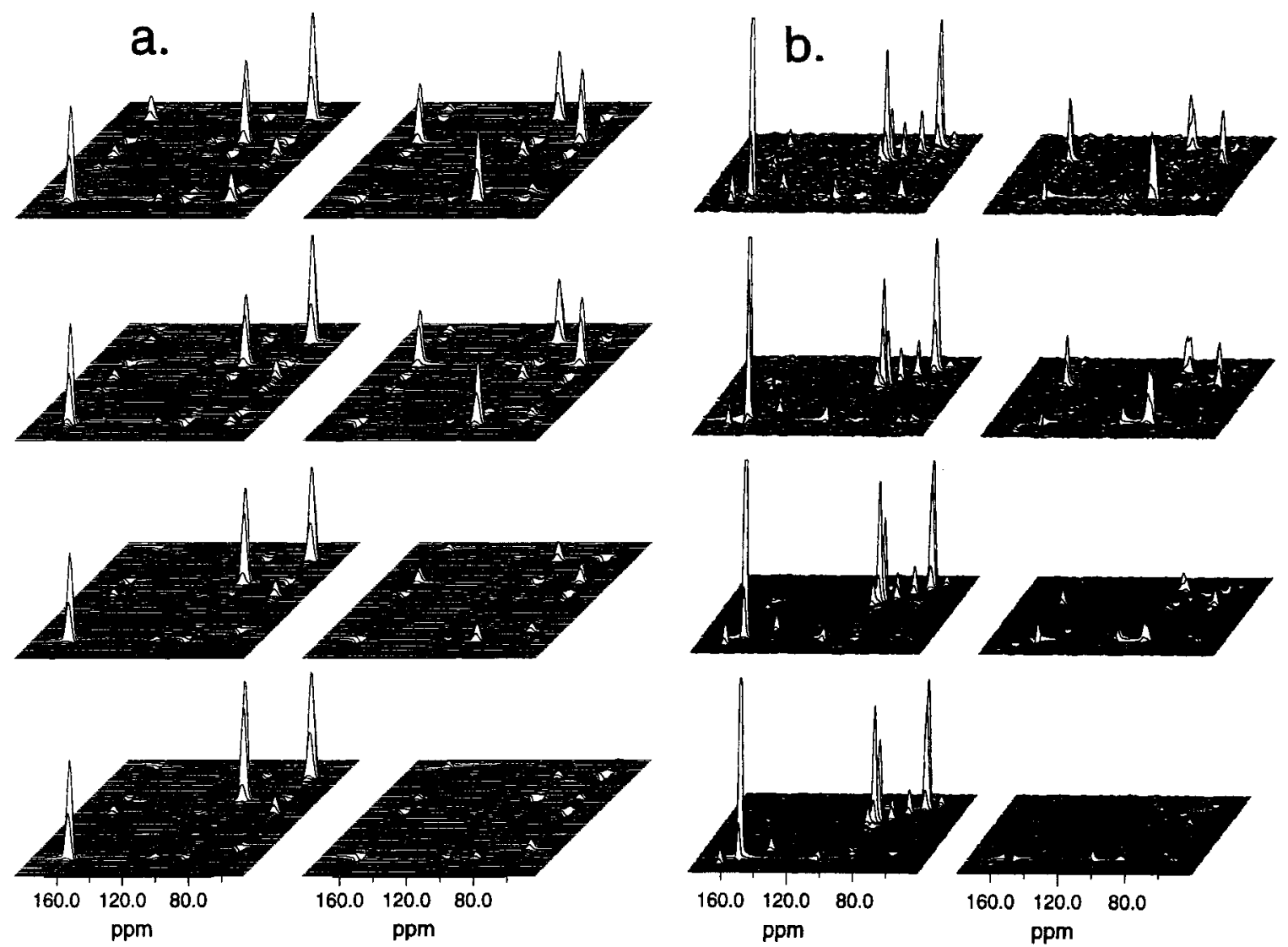

Figure 7. (a) Full 2D matrix simulations of the 2D DRAWS data shown in Figure 7b. To reduce computation times, only $128 \times 128$ data points were simulated for 500 crystallites. Linear prediction followed by zero filling extended the matrix size to $1024 \times 1024$. Positive peaks are shown on the left, with negative peaks from the same spectra on the right. (b) Two-dimensional DRAWS spectra of cytidine-2, $1^{\prime}, 3^{\prime}-{ }^{13} C_{3}$ at different mixing times. Positive peaks are shown on the left and negative peaks from the same spectra on the right. Each spectrum is normalized to the largest positive peak. The mixing times, from top to bottom, are 4.9, 3.3, 1.6, and $0.8 \mathrm{~ms}$, respectively.

In which $I_{y}^{(i)}$ is the $y$ component of the spin $i$ magnetization at the beginning of the mixing period, $\tau_{\mathrm{m}}=0$. After the mixing period, magnetization is detected at other resonances, corresponding to the observed cross-peak between the initial and the detected spins

$$
\begin{aligned}
& \rho_{i}\left(\tau_{m}\right)=U_{m i x} \rho_{i}(0) \bigcup{ }_{m i x}^{\dagger} \\
& s_{i j}\left(\tau_{m}\right)=\operatorname{Tr}\left\{I_{y}^{(j)} \rho_{i}\left(\tau_{m}\right)\right\}
\end{aligned}
$$

In such simulations, the propagator is calculated as for the full matrix simulations, but only over the mixing period, since the chemical shift separation provided by the $t_{1}$ and $t_{2}$ periods is no longer needed. Changing the initial density matrix and the observable in this way allows direct simulation of the crosspeak intensity between resonances $i$ and $j$ as a function of the mixing time, streamlining both the simulation and the data processing. The advantage in reduced computation time is significant, as it allows relaxation effects to be included in simulations of three-spin systems.

For simulating larger spin systems, the number of time increments in the calculation can be reduced further by using an approximate expression for the Hamiltonian to avoid the numerical integration over the mixing period. Since dipolar recoupling sequences are generally cyclic, with a repetition period equal to an integer number of rotor periods, and since the detection of the buildup curve is stroboscopic relative to this cycle time, either average Hamiltonian or Floquet theory can be used to calculate an effective Hamiltonian for the cycle period. If average Hamiltonian theory is used, an effective propagator can be calculated as

$$
U=\exp \left\{-i \tau_{c}\left[\bar{H}^{(0)}+\bar{H}^{(1)}+\bar{H}^{(2)}+\ldots\right]\right\}
$$

where $\bar{H}^{(n)}$ is the $n$th order term in the Magnus expansion of the propagator. The Hamiltonian is often truncated after the first, zeroth-order term, although higher order terms may be included for more accurate results. For DRAWS, the zeroth-order average dipolar Hamiltonian has been calculated as ${ }^{7}$

$$
\begin{aligned}
\bar{H}_{D}^{(0)}=\frac{d_{i j}}{17 \pi}\left\{c_{x x-y y}\left(I_{x}^{(i)} I_{x}^{(j)}-I_{y}^{(i)} I_{y}^{(j)}\right)+\right. \\
\left.c_{z z}\left(3 I_{z}^{(i)} I_{z}^{(j)}-I^{(i)} I^{(j)}\right)\right\}
\end{aligned}
$$

In this expression, $d_{i j}$ is the static dipolar coupling between the two resonances, and the coefficients $c_{x x-y y}$ and $c_{z z}$ depend only on the Euler angles orienting the dipolar tensor in the rotor frame. ${ }^{9}$ For RFDR, the zeroth-order average Hamiltonian expression for the dipolar Hamiltonian has been calculated as

$$
\bar{H}_{D}^{(0)}=-\frac{1}{2} \bar{d}_{i j}\left\{I_{+}^{(i)} I_{-}^{(j)}+I_{-}^{(i)} I_{+}^{(j)}\right\}
$$

in which the coefficient, $\bar{d}_{i j}$, depends on the static dipolar coupling, the dipolar angles, the rotor speed, and the isotropic chemical shift difference between the two resonances. ${ }^{2}$

Using average Hamiltonian expressions reduces computational time, and often simplifies the calculation by removing any explicit dependence on the CSA tensor or its orientation in the crystal frame. Unfortunately, removal of the CSA depen- 
dence is an approximation that is not always warranted. In the DRAWS experiments applied to 2-spin systems, when chemical shift anisotropy is neglected, the cross-peak buildup curves based on numerically integrated Hamiltonians are nearly identical to those obtained with the approximate Hamiltonian calculated by zeroth-order average Hamiltonian theory. When chemical shift terms are included in the numerical simulations, some differences become apparent, but they remain small, particularly at short mixing times. In the RFDR experiments, however, the chemical shift tensor values and their relative orientations in the crystal frame are important experimental parameters, and neglecting these terms from the effective Hamiltonian does not yield simulated cross-peak buildup curves that agree with the experimental data obtained from known systems.

Simulations Methods. We have compared the experimental cross-peak buildup curves obtained from the doubly and triply labeled cytidine samples by the 2D DRAWS and 2D RFDR techniques with simulated results using the three simulation methods described above: full matrix simulations, cross-peak buildup simulations, and approximate cross-peak buildup simulations. The full matrix simulations were carried out with FORTRAN code optimized for MAS calculations on a Dec Alpha 3000 running at $180 \mathrm{MHz}$. For the 2-spin system, without relaxation, calculation of a $128 \times 128$ 2D DRAWS data matrix for 500 crystallites required $1 \mathrm{~h}$ of CPU time. For a 3-spin system, the same calculation took $12 \mathrm{~h}$. In data processing, the matrix size was expanded to $256 \times 256$ by linear prediction and zero filled to a final matrix size of $1024 \times 1024$. The simulated data were Fourier transformed to yield 2D spectra, as shown in Figure $7 \mathrm{~B}$ for cytidine-2, $1,{ }^{\prime} 3^{\prime}-{ }^{13} C_{3}$. The volumes of the cross-peaks in these simulated matrices were quantified with the same methods used to treat the experimental data. Parameters used in the simulations are given in Table 1.

Two-spin DRAWS simulations, which included relaxation effects, assumed 2000 crystallite orientations. The computation of a 10 point DRAWS dephasing curve required 5 min on a Pentium 300. Both the numerical and the approximate crosspeak buildup curve simulations for 3-spin systems were run using code written in Matlab 5.0 $0^{31}$ running (uncompiled) on either a PowerMac 6500 or a Gateway Pentium Pro running at $200 \mathrm{MHz}$. For a 3-spin system, a 2000 crystallite simulation based on the numerically integrated Hamiltonian required 90 min on the Macintosh. When relaxation is included, the calculation requires $5 \mathrm{~h}$. The approximate cross-peak buildup curves were also run on the Macintosh. They required $1.5 \mathrm{~min}$ for a 2 -spin system (500 crystallites), and $10 \mathrm{~min}$ for a 3 -spin system.

Simulation and Analysis of 2D Data for the 2-Spin Systems: Cross-peak buildup curves for cytidine-2, $1^{\prime}-{ }^{13} C_{2}$ and cytidine- $2,2^{\prime}-{ }^{13} C_{2}$ were obtained with both 2 D DRAWS and 2D RFDR, and distance measurements from these experiments were compared with the results obtained from the one-dimensional methods. To obtain the cross-peak buildup curves, five matrices of 5125128 points each were collected for each sample. For the cytidine- $2,1^{\prime}-{ }^{13} C_{2}$ sample, mixing times of $1,2,4,6$, and 8 cycles of dipolar recoupling were used, while for the longer internuclear distance in cytidine- $2,2^{\prime}-{ }^{13} C_{2}$ mixing times of 2,6 , 10,12 , and 16 cycles were used. Quantitation of the twodimensional experiments is discussed below.

For cytidine-2, $2^{\prime}-{ }^{13} C_{2}$ comparison of the experimental data with simulations is shown in Figure 8. The full 2D matrix simulations (open markers) fit the 2D DRAWS experimental data (filled markers) quite well. The cross-peak buildup simulations (solid lines) also fit the experimental data quite closely,

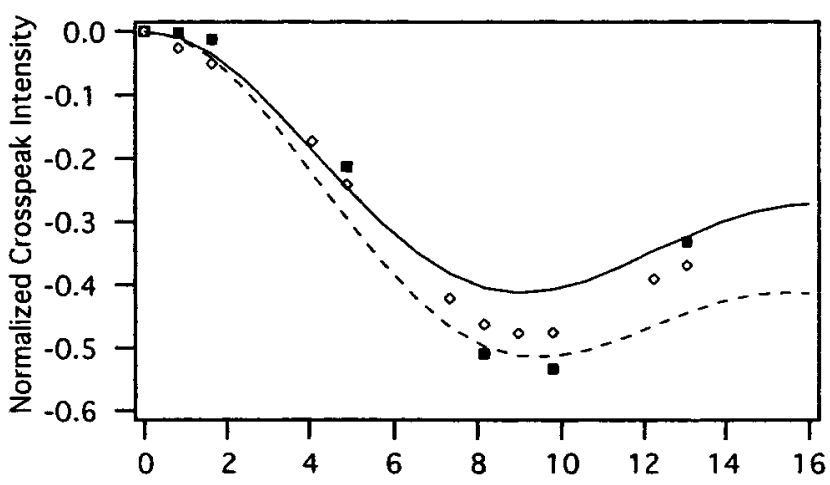

Figure 8. Simulated and experimental 2D DRAWS cross-peak buildup curves obtained from cytidine- $2,2^{\prime}-{ }^{13} C_{2}$ samples. The experimental data are shown with solid squares. Full 2D matrix simulations are shown with open diamonds. One-dimensional cross-peak buildup simulations based on numerical integration of the Hamiltonian are shown with solid lines, while those based on average Hamiltonian expressions are shown with dashed lines. Fits to the other two buildup curve data sets from the doubly labeled samples are qualitatively similar.

although slight differences appear in the maximum cross-peak intensities. These are probably due to the effects of the sideband suppression sequences that are included in the full 2D matrix simulations, but not in the cross-peak buildup simulations. The cross-peak buildup simulations based on average Hamiltonian theory are indicated by dashed lines. For 2D DRAWS, these calculations agree quite well with the experimental data, particularly at short mixing times. Both the initial slope of the buildup curves and the timing of the cross-peak maxima are quite close to that obtained from exact simulations. Based on these results, reasonably accurate distance measurements should be feasible using this relatively rapid simulation method.

In general, the cross-peak buildup simulations that utilize the average Hamiltonian given in eq 8 do not reproduce the 2D RFDR experimental data effectively. This is probably due to the absence of chemical shift parameters in the average Hamiltonian expression and could therefore be corrected by higher order calculations that included chemical shift anisotropies and their relative tensor orientations in the crystal frame. Such calculations are beyond the scope of the current study, and so cross-peak buildup simulations based on the average Hamiltonian in eq 8 were not pursued. The experimental buildup curves are reproduced by the more exact numerical cross-peak buildup calculations.

Variation of the dipolar couplings in the 2D simulations of the doubly labeled cytidine systems yields distance measurements in close agreement with the 1D DRAWS result. The uncertainty is slightly larger due to the small number of data points used to define the curve and is estimated as $\pm 0.2 \AA$.

Simulation and Analysis of 2D Data for the 3-Spin Systems. In Figure 9, experimental 2D DRAWS and 2D RFDR cross-peak buildup curves for the triply labeled samples cytidine$2,1^{\prime}, 3^{\prime}-{ }^{13} C_{3}$, and cytidine- $2,2^{\prime}, 5^{\prime}-{ }^{13} C_{3}$ are compared to simulations. Parts a and c of Figure 9 compare 2D DRAWS data for cytidine- $2,1^{\prime}, 3^{\prime}-{ }^{13} C_{3}$ and cytidine- $2,2^{\prime}, 5^{\prime}-{ }^{13} C_{3}$, respectively, to three types of simulations: full numerical matrix simulations (open markers), numerical simulations of cross-peak buildup curves (solid lines), and spectral simulations based on average Hamiltonian theory (dashed lines). Parts $b$ and $d$ of Figure 9 compare 2D RFDR data for cytidine- $2,1^{\prime}, 3^{\prime}-{ }^{13} C_{3}$, and cytidine$2,2^{\prime}, 5^{\prime}-{ }^{13} C_{3}$, respectively, to numerical simulations of cross-peak buildup curves only. In Figure 9a (and in Figure 9c), experimental data points are indicated by closed markers, i.e., C2$\mathrm{C}^{\prime}$ data are represented by inverted, closed triangles, $\mathrm{Cl}^{\prime}-$ 
a.

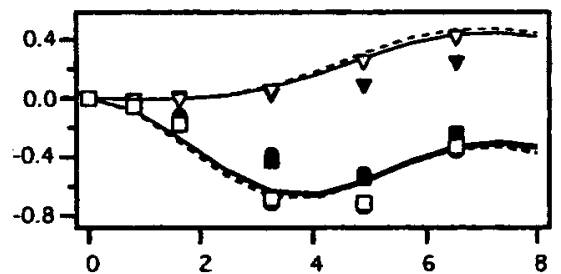

b.

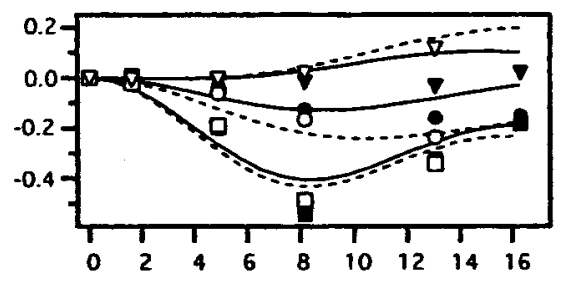

C.

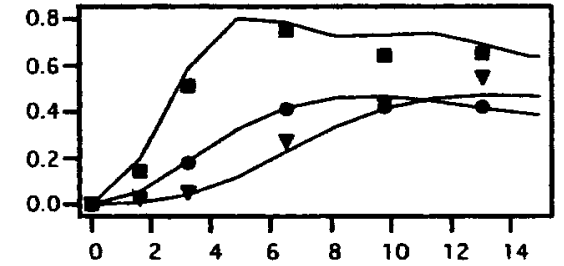

d.

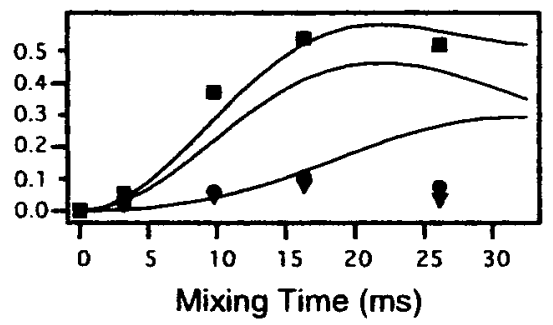

Figure 9. Cross-peak buildup curves obtained from triply labeled cytidine samples. The curves in (a) and (c) were obtained from cytidine$2,1^{\prime}, 3^{\prime}-{ }^{13} C_{3}$ with 2D DRAWS and 2D RFDR, respectively. The curves in (b) and (d) were obtained from cytidine- $2,2^{\prime}, 5^{\prime}-{ }^{13} C_{3}$ with 2D DRAWS and 2D RFDR, respectively. One-dimensional cross-peak buildup simulations of 2D DRAWS and 2D RFDR data based on numerical integration of the Hamiltonian are shown with solid lines. Simulations of 2D DRAWS data based on average Hamiltonian calculations are shown with dashed lines. Full matrix simulations of 2D DRAWS data are shown with open markers. The experimental data are shown with filled markers. In (a) and (c) the inverted triangles are $\mathrm{C} 2-\mathrm{C}^{\prime}$ distance measurements/simulations, the circles are $\mathrm{C1}^{\prime}-\mathrm{C} 3^{\prime}$ distance measurements/simulations, and the squares are $\mathrm{C} 2-\mathrm{C}^{\prime}$ distance measurements/ simulations. In (b) and (d) inverted triangles are $\mathrm{C} 2-\mathrm{C} 5^{\prime}$ distances measurements/simulations, the squares are the $\mathrm{C} 2-\mathrm{C} 2$ ' distance measurements/simulations, and the circles are the $\mathrm{C} 2^{\prime}-\mathrm{C} 5^{\prime}$ distance measurements/simulations.

$\mathrm{C}^{\prime}$ data by closed circles, and $\mathrm{C} 2-\mathrm{C}^{\prime}$ by closed squares. As shown in Figure 9a, C2-C3' cross-peaks are not observed for short DRAWS mixing times, but appear as small, positive crosspeaks at mixing times longer than $2 \mathrm{~ms}$. The $\mathrm{C} 2-\mathrm{C} 3^{\prime}$ crosspeak intensity is therefore dominated by indirect transfers, probably involving the $\mathrm{Cl}^{\prime}$ nucleus. This interpretation is shown to be reasonable by all three types of simulations, each of which calculates the net $\mathrm{C} 2-\mathrm{C}^{\prime}$ intensity for the $\mathrm{C} 2-\mathrm{Cl}^{\prime}-\mathrm{C}^{\prime}$ system. In contrast, the $\mathrm{C} 2-\mathrm{Cl}^{\prime}$ and $\mathrm{Cl}^{\prime}-\mathrm{C}^{\prime}$ data are negative cross-peaks, indicating that intensities of these cross-peaks are dominated by direct coherence transfers. The experimental $\mathrm{C} 2-$ $\mathrm{C1}^{\prime}$ and $\mathrm{C1}^{\prime}-\mathrm{C}^{\prime}$ data sets virtually superimpose, demonstrating that DRAWS suppresses chemical shifts very effectively.

In Figure 9b (and in Figure 9d) $\mathrm{C} 2-\mathrm{C} 5$ ' data derived from cytidine- $2,2^{\prime}, 5^{\prime}-{ }^{13} C_{3}$ are shown as inverted, closed triangles, $\mathrm{C} 2^{\prime}-\mathrm{C} 5^{\prime}$ data are closed circles, and $\mathrm{C} 2-\mathrm{C} 2^{\prime}$ data are closed squares. The $\mathrm{C} 2$ and $\mathrm{C}^{\prime}$ nuclei are separated by $5.5 \AA$ in the crystal structure, and although simulations indicate that positive $\mathrm{C} 2-\mathrm{C}^{\prime}$ cross-peaks should appear at mixing times longer than
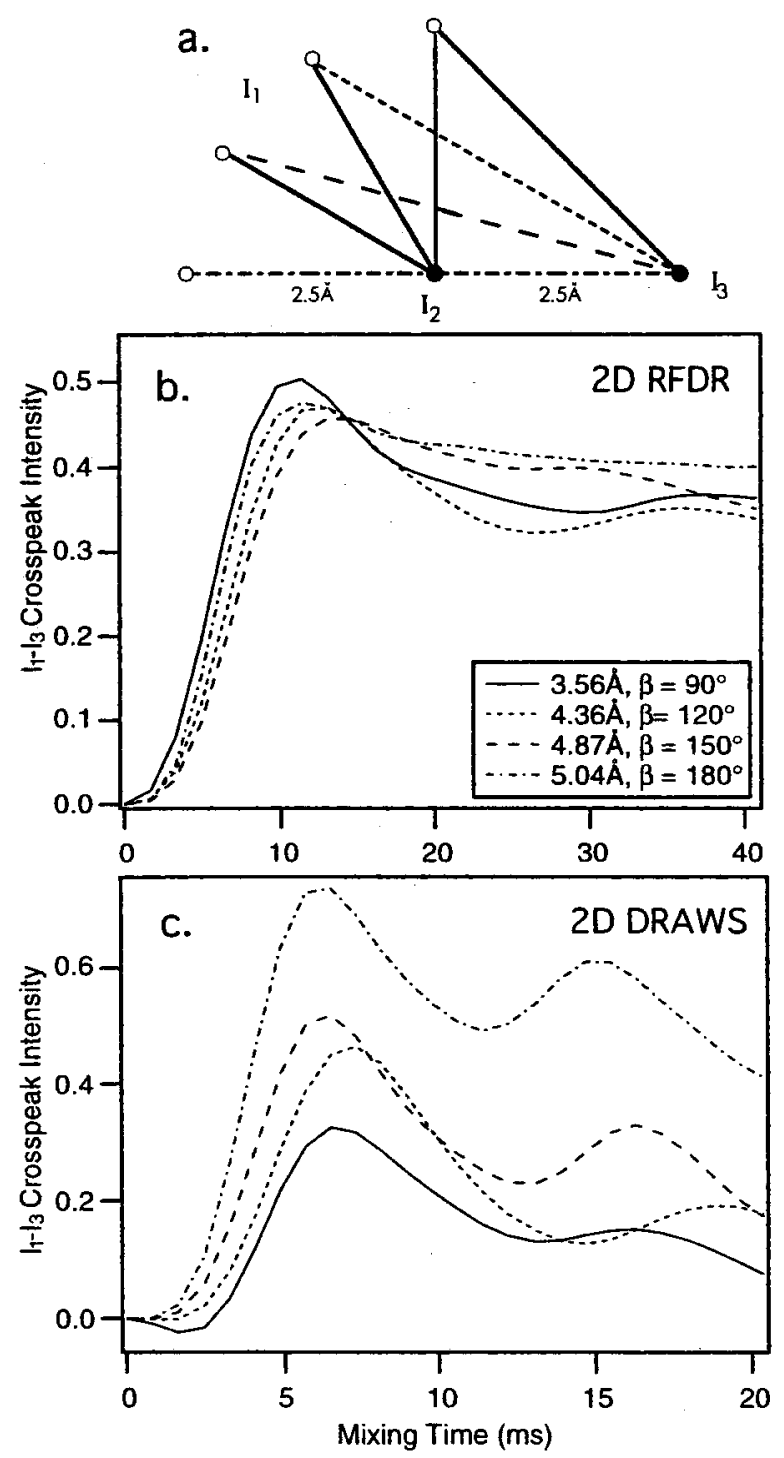

Figure 10. Effect of spin system geometry on distance measurements in a 3 -spin system. Simulation parameters correspond to the $\left[2,1^{\prime}, 3^{\prime}-\right.$ ${ }^{13} \mathrm{C}_{3}$ ]cytidine sample in which the $\mathrm{C} 2$ nucleus is $\mathrm{I}_{1}, \mathrm{Cl}^{\prime}$ is $\mathrm{I}_{2}$, and $\mathrm{C}^{\prime}$ is $\mathrm{I}_{3}$.

$8 \mathrm{~ms}$, experimental sensitivity was not high enough to observe these transfers in 2D DRAWS experiments. The $\mathrm{C} 2-\mathrm{C}^{\prime}$ and $\mathrm{C} 2^{\prime}-\mathrm{C}^{\prime}{ }^{\prime}$ nuclei are closer (3.2 and $3.7 \AA$, respectively, in the crystal structure) and their negative cross-peaks appear in $2 \mathrm{D}$ DRAWS spectra at short mixing times and agree well with simulations.

As shown in Figure 9c, cross-peak buildup simulations (using internuclear distances derived from the crystal structure and CSA parameters from model compounds, described above) fit the experimental RFDR cross-peak buildup data for cytidine-2, $1,3^{\prime}$ ${ }^{13} C_{3}$ nearly as well as in the 2 -spin case. Although cross-peak buildup simulations fit the $\mathrm{C} 2-\mathrm{C} 2^{\prime}$ data in Figure $9 \mathrm{~d}$ almost perfectly, similar simulations did not fit the $\mathrm{C} 2-\mathrm{C}^{\prime}$ or the $\mathrm{C} 2^{\prime}-$ C5' data.

Although all of the 2D DRAWS data and most of the 2D RFDR data for cytidine- $2,1^{\prime}, 3^{\prime}-{ }^{13} C_{3}$, and cytidine- $2,2^{\prime}, 5^{\prime}-{ }^{13} C_{3}$ can be simulated, the degree to which these data are sensitive to individual internuclear distances must be considered. This is an especially important issue for cross-peaks with intensity deriving in part from indirect coherence transfers. The effect of indirect coherence transfers in the cytidine- $2,1^{\prime}, 3^{\prime}-{ }^{13} C_{3}$ system can be seen in the simulations shown in Figure 10. In these 

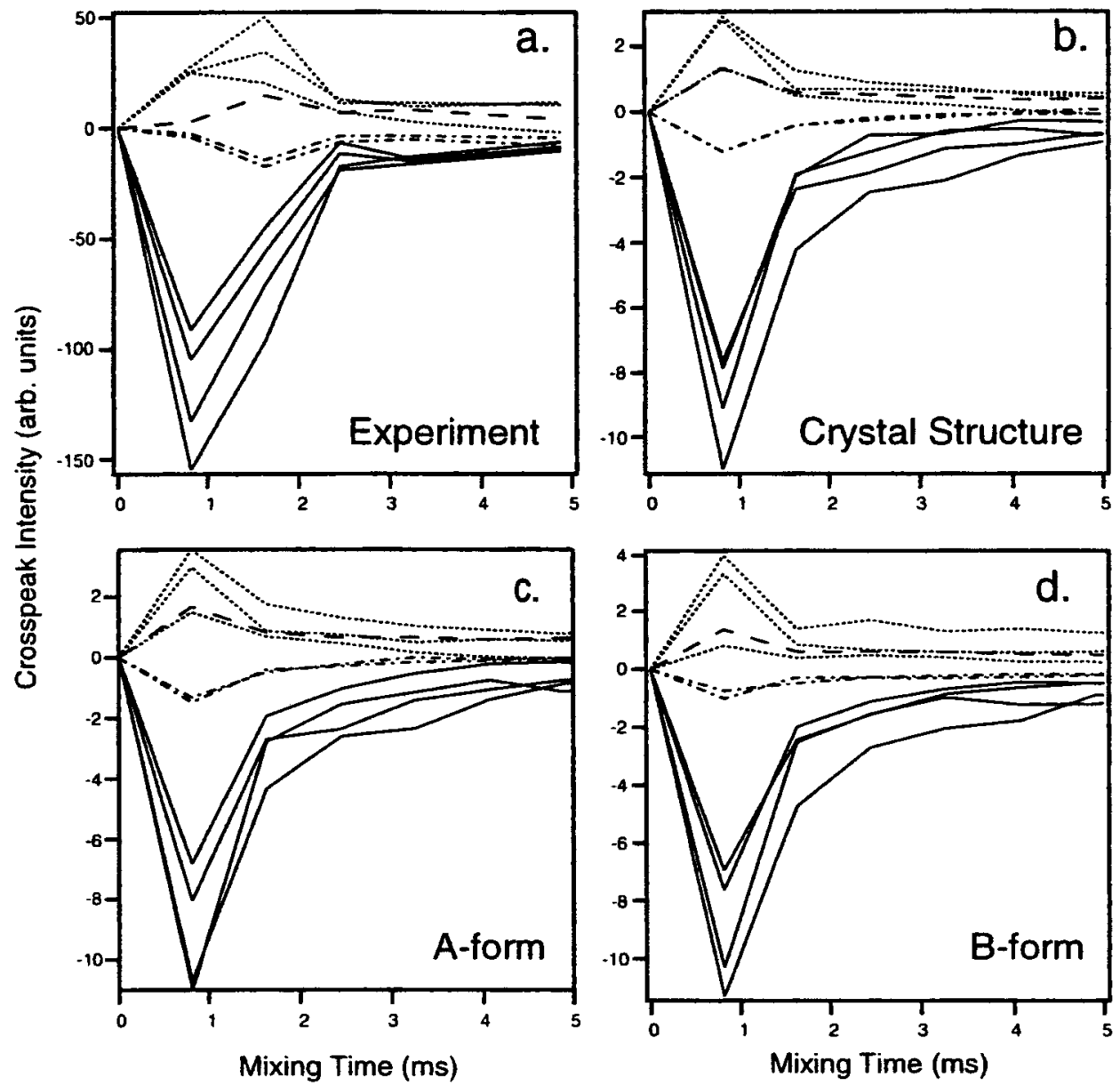

Figure 11. Experimental and simulated cross-peak buildup curves of the 5-spin sugar ring region of uniformly labeled cytidine. Simulations were based on the DRAWS zeroth-order average Hamiltonian expression. Solid lines represent one-bond distances (i.e., $\mathrm{C1}^{\prime}-\mathrm{C} 2^{\prime}, \mathrm{C} 2^{\prime}-\mathrm{C} 3^{\prime}$, etc). Dotted lines represent two-bond distances (i.e., $\mathrm{C} 1^{\prime}-\mathrm{C} 3^{\prime}$, etc). Dash dot lines correspond to three-bond distances (i.e., $\mathrm{C} 2^{\prime}-\mathrm{C}^{\prime}$ and $\left.\mathrm{C} 1^{\prime}-\mathrm{C} 4^{\prime}\right)$. The dashed line corresponds to the four-bond distance, $\mathrm{C1}^{\prime}-\mathrm{C}^{\prime}$. The un-normalized experimental cross-peak intensities are shown in (a). The simulations in (b) -(d) correspond to the crystal structure, ideal A-form, and ideal B-form DNA sugar ring structures, respectively. Each simulation includes 2000 crystallites.

simulations, the $\mathrm{C} 2-\mathrm{C}^{\prime}-\mathrm{C} 3^{\prime}$ angle is varied while the $\mathrm{C1}^{\prime}-$ $\mathrm{C} 3^{\prime}$ and $\mathrm{C} 1^{\prime}-\mathrm{C} 2$ distances are held constant at $2.5 \AA$, thus varying the $\mathrm{C} 2-\mathrm{C}^{\prime}$ distance from 3.5 to $5.1 \AA$. The simulated 2D RFDR cross-peak buildup curves shown in Figure $10 \mathrm{~b}$ are nearly unchanged, suggesting that these molecular conformations cannot be distinguished with this technique under these experimental conditions. At the bottom of the figure, the 2D DRAWS buildup curves do vary with the conformational change; however, the variation is not in the timing of the cross-peak maximum, as would be expected for a distance change, but rather in the maximal intensity. The intensity increases in the positive direction with increased internuclear distance between the $\mathrm{C} 2$ and $\mathrm{C} 3^{\prime}$ moieties, corresponding to a decrease in the influence of the direct coherence transfer pathway. Unfortunately, such an effect cannot be readily distinguished from relaxation. Considering these simulations, the precision of the long distance measurements in the triply labeled cytidine samples is estimated to be $\pm 0.5 \AA$.

Simulation and Analysis of 2D Data for the Uniformly Labeled (i.e., 9-Spin) Sample. In analyzing the experimental cross-peak buildup curves obtained from the uniformly labeled cytidine sample (i.e., a $9{ }^{13} \mathrm{C}$ spin system), only simulations of cross-peak buildup curves using average Hamiltonian theory were performed. General, an $n$-spin computer code based on the DRAWS average Hamiltonian expression has been written in Matlab 5.0. ${ }^{31}$ Calculation of the full 9-spin system requires
50 min for each crystallite on a Gateway Pentium Pro $200 \mathrm{MHz}$ computer. A 7-spin system required $8.4 \mathrm{~min} /$ crystallite on the same platform. Since an important question in these studies is is the degree to which nucleic acid sugar ring conformation can be accurately determined from 2D dipolar recoupling studies of uniformly labeled nucleic acids, we carried out a series of simulations on a 7-spin system consisting of the five sugar ring resonances and the two closest carbon resonances on the base, $\mathrm{C} 2$ and $\mathrm{C} 6$.

The 2D DRAWS experimental and simulated cross-peak buildup curves for the sugar ring resonances are compared in Figure 11. There is relatively close agreement between the simulated and experimental data. To determine whether this agreement was sufficient to differentiate between different sugar ring conformations, simulations were also carried out on the basis of ideal A-form and ideal B-form cytidine structures. These simulations are shown in Figure 11c,d, respectively. It is clear that, although the agreement between the simulated and experimental data is quite good, the differences between the simulations corresponding to different sugar ring conformations are very small. Simulation of the experimental cross-peak buildup curves thus does not indicate a particular sugar ring conformation.

Phenomenological Analyses of 2D Data for Uniformly Labeled Cytidine (i.e., 9 Spins). In solution state NMR, structures are derived from many relatively imprecise distance 

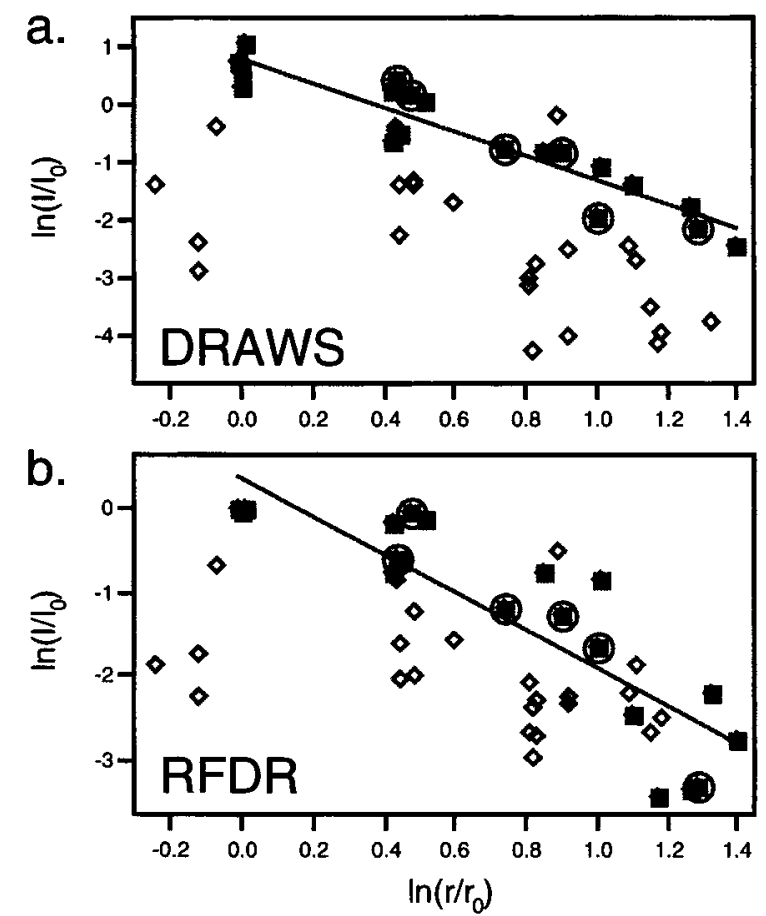

Figure 12. Correlation plots between cross-peak intensity ratios and crystallographic internuclear distance ratios for 2D DRAWS (a) and 2D RFDR (b) following eq 11. Cross-peak data obtained from uniformly labeled cytidine that were included in the $\chi^{2}$ calculation are shown as filled squares. C5 and C6 yielded low-intensity signals. Therefore, crosspeaks involving C5 and C6 were of very low intensity and thus were not included in the calculation of the correlation function. These data are shown as open diamonds. Circled data are derived from triply labeled cytidine samples and were included in the calculation of the correlation function with the same weighting as the data derived from uniformly labeled cytidine. In each plot the straight line is a leastsquares fit to the data.

constraints in combination with distance geometry and energy minimization techniques. Solution state 2D NOESY data are analyzed by a number of different methods. The most common method is to roughly characterize the cross-peak intensities as strong, medium, or weak and associate distance ranges with each classification. Alternatively, the time behavior of the crosspeaks may also be considered, usually by determining the slope of the initial cross-peak buildup curve. It has been proposed that similar methods are appropriate for analysis of solid state NMR dipolar recoupling buildup curves from uniformly labeled samples. ${ }^{32}$ We have therefore applied both of these approaches to the 2D RFDR and 2D DRAWS buildup curves obtained from the uniformly ${ }^{13} \mathrm{C}$ and ${ }^{15} \mathrm{~N}$ labeled cytidine sample. In ref 10 we showed that in a phase-sensitive two-dimensional DRAWS spectrum the cross-peak intensity has the form

$$
I(\tau) \propto \sin (\beta \tau) \sin \left(\frac{(\alpha-\beta) \tau}{2}\right)
$$

where $\alpha=(d / 17 \pi) c_{x x-y y}, \beta=(d / 17 \pi) c_{z z}$, and $d=\left(\gamma^{2} \hbar / r^{3}\right.$. In the limit that $\alpha \tau$ and $\beta \tau$ are much less than $1, \sin (\beta \tau) \approx \beta \tau$, $\sin ((\alpha-\beta) / 2] \tau) \approx(\alpha-\beta) \tau / 2$, and the cross-peak intensity in a 2D DRAWS spectrum has the form

$$
I(\tau) \propto \frac{\alpha \beta-\beta^{2}}{2} \tau^{2} \approx \frac{d^{2} \tau^{2}}{578 \pi^{2}}\left(c_{z z} c_{x x-y y}-c_{z z}{ }^{2}\right)
$$

Equation 10 means that at a given mixing time $\tau$ the 2D DRAWS cross-peak intensity depends on $1 / r{ }^{6}$ An internuclear
TABLE 2: Cytidine Dipolar Simulation Parameters

\begin{tabular}{lccc}
\hline coupling & distance $(\AA)$ & $\theta(\operatorname{deg})^{a}$ & $\phi(\operatorname{deg})^{b}$ \\
\hline $\mathrm{C} 2-\mathrm{C1}^{\prime}$ & 2.519 & 43.2 & 232.1 \\
$\mathrm{C}^{\prime} 1^{\prime}-\mathrm{C} 3^{\prime}$ & 2.524 & 101.1 & 275.5 \\
$\mathrm{C} 2-\mathrm{C} 3^{\prime}$ & 4.200 & 71.3 & 243.4 \\
$\mathrm{C} 2-\mathrm{C} 2^{\prime}$ & 3.179 & 70.35 & 224.2 \\
$\mathrm{C} 2^{\prime}-\mathrm{C} 5^{\prime}$ & 3.721 & 56.1 & 307.6 \\
$\mathrm{C} 2-\mathrm{C} 5^{\prime}$ & 5.53 & 55.3 & 266.7
\end{tabular}

${ }^{a}$ Polar angle of the dipolar vector in the crystal frame. ${ }^{b}$ Azimulthal angle of the dipolar vector in the crystal frame.

TABLE 3: Comparison of Cytidine Distances Measured by X-ray and by 2D RFDR

\begin{tabular}{lcccccc}
\hline & $\begin{array}{c}\text { X-ray } \\
\text { crystal } \\
\text { structure }\end{array}$ & $\begin{array}{c}\text { X-ray } \\
\text { B-form } \\
\text { structure }\end{array}$ & 2-spin & 2D 3-spin & $\begin{array}{c}\text { RFDR } \\
\text { 9-spin }\end{array}$ & $\begin{array}{c}\text { 9-spin } \\
\text { intensities }\end{array}$ \\
\hline $1^{\prime}-3^{\prime}$ & 2.36 & 2.37 & & $2.4 \pm 0.3$ & $1.8 \pm 0.3$ & $2.2 \pm 0.3$ \\
$2-1^{\prime}$ & 2.46 & 2.47 & $2.4 \pm 0.3$ & $2.4 \pm 0.3$ & $2.3 \pm 0.6$ & $2.3 \pm 0.4$ \\
$2-2^{\prime}$ & 3.19 & 3.71 & $3.2 \pm 0.3$ & $3.2 \pm 0.3$ & $3.1 \pm 0.8$ & $3.6 \pm 0.7$ \\
$2^{\prime}-5^{\prime}$ & 3.75 & 3.20 & & $3.8 \pm 0.5$ & $3.2 \pm 0.9$ & $2.8 \pm 0.5$ \\
$2-3^{\prime}$ & 4.16 & 4.82 & & $4.0 \pm 0.8$ & $3.7 \pm 1.1$ & $4.2 \pm 0.9$ \\
$2-5^{\prime}$ & 5.54 & 5.09 & & $5.5 \pm 0.8$ & $7.8 \pm 3.5$ & $5.0 \pm 1.2$
\end{tabular}

TABLE 4: Comparison of Cytidine Distances Mearsured by $X$-ray and by 2D DRAWS

\begin{tabular}{lcccccc}
\hline $\begin{array}{c}\text { X-ray } \\
\text { crystal } \\
\text { name }\end{array}$ & $\begin{array}{c}\text { X-ray } \\
\text { structure }\end{array}$ & $\begin{array}{c}\text { B-form } \\
\text { structure }\end{array}$ & 2-spin & 2D 3-spin & $\begin{array}{c}\text { DRAWS } \\
\text { 9-spin }\end{array}$ & $\begin{array}{c}\text { 9-spin } \\
\text { intensities }\end{array}$ \\
\hline $1^{\prime}-3^{\prime}$ & 2.36 & 2.37 & & $2.4 \pm 0.3$ & $1.8 \pm 0.3$ & $1.4 \pm 0.3$ \\
$2-1^{\prime}$ & 2.46 & 2.47 & $2.4 \pm 0.2$ & $2.4 \pm 0.3$ & $2.0 \pm 0.3$ & $3.1 \pm 0.3$ \\
$2-2^{\prime}$ & 3.19 & 3.71 & $3.2 \pm 0.2$ & $3.2 \pm 0.3$ & $3.1 \pm 0.6$ & $4.1 \pm 1.1$ \\
$2^{\prime}-5^{\prime}$ & 3.75 & 3.20 & & $3.8 \pm 0.5$ & $3.2 \pm 0.6$ & $2.9 \pm 0.7$ \\
$2-3^{\prime}$ & 4.16 & 4.82 & & $4.0 \pm 0.8$ & $5.4 \pm 1.5$ & $5.2 \pm 1.5$ \\
$2-5^{\prime}$ & 5.54 & 5.09 & & & $6.0 \pm 1.7$ & $6.5 \pm 2.2$
\end{tabular}

distance $r$ can be obtained from a cross-peak intensity $I$, given a knowledge of the internuclear distance $r_{0}$ that corresponds to a second cross-peak intensity $I_{0}$. Assuming the cross-peak intensities were measured at identical mixing times, it is easy to show that

$$
\log \left(\frac{I}{I_{0}}\right)=-6 \log \left(\frac{r}{r_{0}}\right)
$$

To carry out the intensity analysis, cross-peak volumes corresponding to symmetric peaks (e.g., the $\mathrm{C} 2-\mathrm{C}^{\prime}{ }^{\prime}$ cross-peak and the $\mathrm{C}^{\prime}-\mathrm{C} 2$ cross-peak) were added and normalized to the average of the cross-peak volumes corresponding to single bond interactions (i.e., $\left.1^{\prime}-2^{\prime}+2^{\prime}-3^{\prime}+\ldots / n\right)$. This intensity analysis was carried out separately on the cross-peak volumes from each two-dimensional matrix. A linear fit was used to assess the correlation of the observed intensity ratios with the crystal structure distances. Figure 12 shows a $\log -\log$ plot of the cytidine- $U-{ }^{13} C_{9},{ }^{15} N_{3}$ cross-peak intensity ratios $I / I_{0}$ versus the corresponding crystal structure distance ratios $r / r_{0}$ for $2 \mathrm{D}$ DRAWS and 2D RFDR data obtained after $2.448 \mathrm{~ms}$ of dipolar recoupling. The correlation coefficients were $R=-0.9417$ and $R=-0.8801$ for the 2D DRAWS and 2D RFDR results, respectively (where the negative sign in the correlation coefficient corresponds to a negative slope in the correlation plot). ${ }^{33,34}$ Therefore the logarithm of the NMR intensity ratios derived by 2D DRAWS and 2D RFDR show linear a correlation with the logarithm of distance ratios $r / r_{0}$, albeit not a strong one in either case.

The correlation coefficient and the linear fitting equation can be used to "predict" distances and errors from the experimentally observed intensity ratios. Although this analysis cannot be used on experimental data for which the distances are unknown, it 
does yield a measure of the accuracy of this method for distance measurement. If the linear correlation is very good, the distance measurements will be both accurate and precise. For the six cross-peaks also measured in the selectively labeled cytidine samples, distances and error bars derived from the correlations shown in Figure 12 are given in Tables 3 and 4 for DRAWS and RFDR, respectively.

To ensure the validity of the linear approximation used to obtain eq 10, internuclear distances were correlated with the slopes of the cross-peak buildup curves obtained from 2D RFDR and 2D DRAWS at short mixing times. In analogy to eq 11, ratios of initial slopes of cross-peak buildup curves obtained from 2D RFDR and/or 2D DRAWS spectra are equated to ratios of distances:

$$
\log \left(\frac{\mathrm{d} I / \mathrm{d} t}{\mathrm{~d} I_{0} / \mathrm{d} t}\right)=-6 \log \left(\frac{r}{r_{0}}\right)
$$

As before, eq 12 assumes the slopes $\mathrm{d} I / \mathrm{d} t$ and $\mathrm{d} I_{0} / \mathrm{d} t$ are evaluated at identical mixing times. To carry out the initial crosspeak slope analysis, the cross-peak volumes were first normalized to the sum of the corresponding auto-peak volumes. The cross-peak volumes corresponding to the linear part of the initial buildup curve, $(\mathrm{d} I / \mathrm{d} t)_{\text {initial}}$, were then evaluated as ratios, plotted in $\log -\log$ format, and fit to a straight line according to eq 12 . The resulting correlation plots for the 2D RFDR and 2D DRAWS data are shown in Figure 13. The coefficient of linear correlation for the 2D RFDR data was found to be about $R=$ -0.95 and $R=-0.92$ for the 2D DRAWS data. Internuclear distances were also evaluated from the linear correlation plots in Figure 13, and are shown in Tables 3 and 4.

\section{Discussion}

Numerical simulations indicate that very precise and accurate internuclear distances can be obtained from doubly and triply labeled cytidine samples, especially for distances less than 3.1$3.5 \AA$. In doubly labeled cytidines, treating both the internuclear distance and $R_{2 \mathrm{dq}}$ as adjustable parameters in least squares calculations does appear to degrade somewhat the precision with which internuclear distances can be determined, but relative uncertainties of $10 \%$ or less are possible to achieve for internuclear distances less than 3.1-3.5 $\AA$. It should be noted that Heller et al. ${ }^{12}$ have shown that in rotational resonance experiments performed on inhomogeneously broadened systems, the correlation between internuclear distance and $R_{2 \mathrm{zq}}$ appears to increase with internuclear distance and thus the uncertainty of long distance measurements is markedly degraded. The effect that correlation of $r(i, j)$ and $R_{2 \mathrm{dq}}$ has on the precision of long distance (i.e., >3.1 A) DRAWS measurements in doubly labeled, crystalline systems will be addressed elsewhere.

Although values for both the internuclear distance and $R_{2 \mathrm{dq}}$ were necessary to fit 1D DRAWS decay curves of doubly labeled cytidines, and the internuclear distance and $R_{2 \mathrm{zq}}$ were similarly necessary to fit $1 \mathrm{D}$ RFDR data, neither $R_{2 \mathrm{dq}}$ nor $R_{2 \mathrm{zq}}$ were required to fit the 2D DRAWS or 2D RFDR cross-peak buildup curves, respectively. This is attributed to the effects of normalizing the cross-peak volumes to the auto-peak volumes at each point in the buildup curve. In the 3-spin systems, this normalization technique is not sufficient to remove the effects of relaxation, and the fit between the experimental and simulated results is compromised. Neither $R_{2 \mathrm{dq}}$ nor $R_{2 \mathrm{zq}}$ values, derived from 1D fits, can be included in cross-peak buildup curve simulations even for the three spin systems. When compared to the unnormalized cross-peak volumes, this method does yield

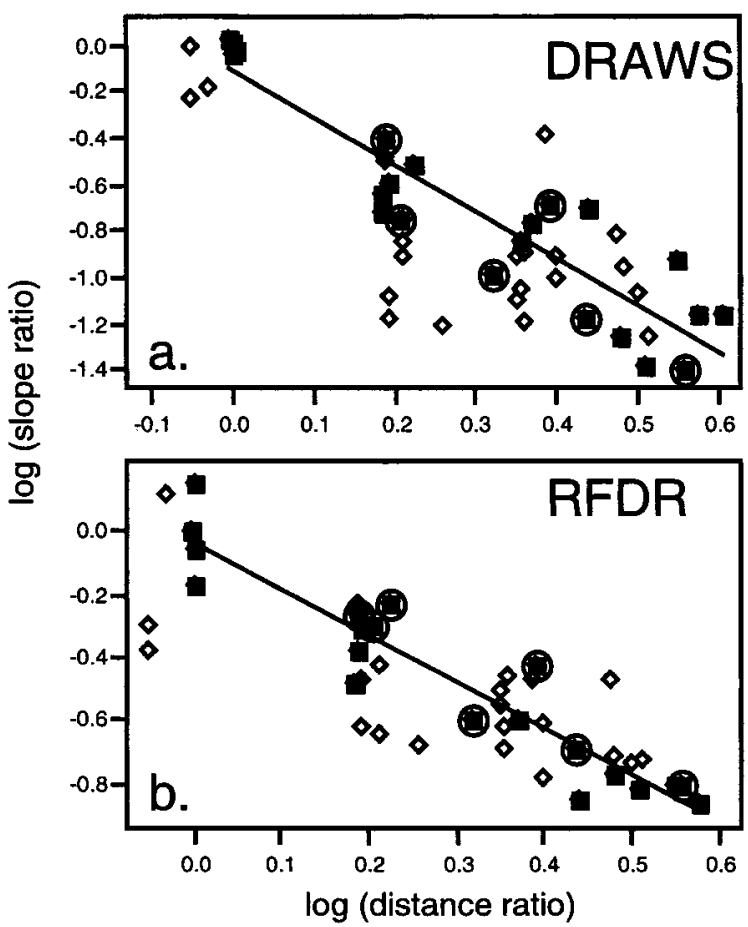

Figure 13. Correlation plots between the slopes obtained from the initial cross-peak buildup curves and crystallographic internuclear distance ratios, according to eq 12, for 2D DRAWS (a) and 2D RFDR (b). Conventions for data symbols are the same as in Figure 12. Each cross-peak was normalized to the sum of the corresponding auto-peaks. To obtain the slope, the initial (linear) region of the buildup curve was fit with a straight line through the origin.

somewhat improved fits, particularly for the later time points. The apparent scatter is significantly worse, however, in the unnormalized data since there is no correction for spectrometer variations over the course of these long experiments. In the present work the precision and accuracy of long distances determined by 2D DRAWS appears to be limited by a number of other effects including indirect transfers. As discussed above, the attempts were made to minimize the effects of $R_{2 \mathrm{dq}}$ in 2D DRAWS experiments by normalization of cross-peak intensities to auto-peak intensities.

From the data summarized in Tables 3 and 4, it is clear that two-dimensional dipolar recoupling techniques can potentially provide many internuclear distance constraints simultaneously from uniformly labeled systems. But unlike distance measurements obtained by recoupling of doubly and triply labeled cytidines, distance mesurements obtained from uniformly labeled cytidine are markedly low in precision and in some cases accuracy. A number of factors contribute to this situation. First, it is difficult to ensure the validity of the linear approximation made in eq 10. Even if this approximation is valid, the simple expression for cross-peak intensity shown in eq 9 assumes spins interact as isolated pairs. This is an excellent approximation in doubly labeled systems, a fair approximation in the triply labeled cytidines, and a poor approximation in the uniformly labeled cytidine. In uniformly labeled cytidine, local spin geometry may complicate the interpretation of cross-peak intensities, as shown in Figure 10, which in turn will invalidate the simple linear correlations proposed in eqs 11 and 12. Deviations from eqs $11 / 12$ are expected to be especially serious for spin pairs that are separated by long distances. In fact, if directly bonded spin pairs are omitted from the correlation coefficient calculations that accompany the data in Figures 12 and 13, the degree of linear correlation is markedly diminished. 


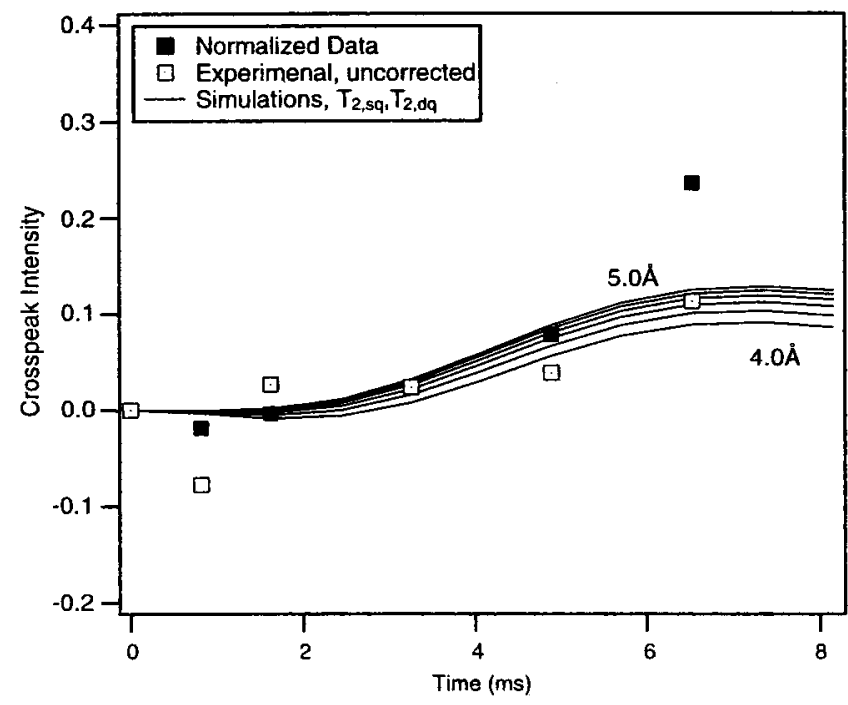

Figure 14. Comparison of experimental and simulated 2D DRAWS buildup curves for the $2-3^{\prime}$ cross-peak in crystalline cytidine-2, $1^{\prime}, 3^{\prime}$ ${ }^{13} C_{3}$. Uncorrected data were normalized to the total auto-peak intensity in the first experiment. The "normalized" data were further corrected for relaxation by dividing cross-peak volumes by auto-peak volumes at each point in the buildup curve. Simulation parameters are listed in Tables 1 and 2 .

Nevertheless, it is interesting to consider the degree of structural detail that can be obtained from the data given in Tables 3 and 4. For example, in solution NMR the structure of the furanose rings in DNA is determined by NOE measurement of proton-proton distances ${ }^{35}$ and by estimation of torsion angles from scalar coupling data. ${ }^{36}$ Several proton-proton distances can be easily obtained from NOE measurements and are diagnostic of furanose ring structure. For example, in A-form DNA, the $\mathrm{H} 2^{\prime \prime}-\mathrm{H} 4^{\prime}$ distance is $2.35 \AA$, while this changes to $3.8 \AA$ in the $\mathrm{B}$-form. The $\mathrm{H} 1^{\prime}-\mathrm{H} 4^{\prime}$ distance changes from 3.3 to $2.5 \AA$. Barring complications arising from spin diffusion, such distance changes are, in principle, well within the capabilities of NOE measurements. In contrast, one of the largest changes in ${ }^{13} \mathrm{C}-{ }^{13} \mathrm{C}$ distances in these two conformations is the $\mathrm{C} 2-$ C2' distance, which changes from $3.2 \AA$ in the A-form to $3.7 \AA$ in the $\mathrm{B}$-form. Consequently, relatively precise $\mathrm{C}-\mathrm{C}$ distance measurements are required to define the sugar ring conformation, and the local structure in general. While many, rough distance constraints are obtained from the two-dimensional solid state dipolar recoupling experiments on the uniformly labeled sugar ring, as shown in Tables 3 and 4, many of these constraints are clearly insufficient to determine the pucker of the sugar ring.

In studies of selectively labeled materials, the precision of the distance measurements is considerably improved, even in systems with moderate amounts of indirect coherence transfer. Such measurements do allow determination of the sugar ring pucker, though only in some cases. In cytidine- $2,1^{\prime}, 3^{\prime}-{ }^{13} C_{3}$ only the long, $2-3^{\prime}$ distance changes between sugar ring conformations: $4.2 \AA$ in A-form DNA vs $4.8 \AA$ in B-form DNA. As demonstrated by the simulations in Figure 14, the 2D DRAWS data do not allow differentiation between the A-form and B-form structures. By contrast, in cytidine- $2,2^{\prime}, 5^{\prime}-{ }^{13} C_{3}$, all the internuclear distances change by similar, relatively large amounts. As shown in Figure 15, these coupled changes allow differentiation of the A-form and B-form structures based on the experimental 2D DRAWS buildup curves. This differentiation is clearly based on the two shorter distances that exhibit negative cross-peaks in the 2D DRAWS experiments.
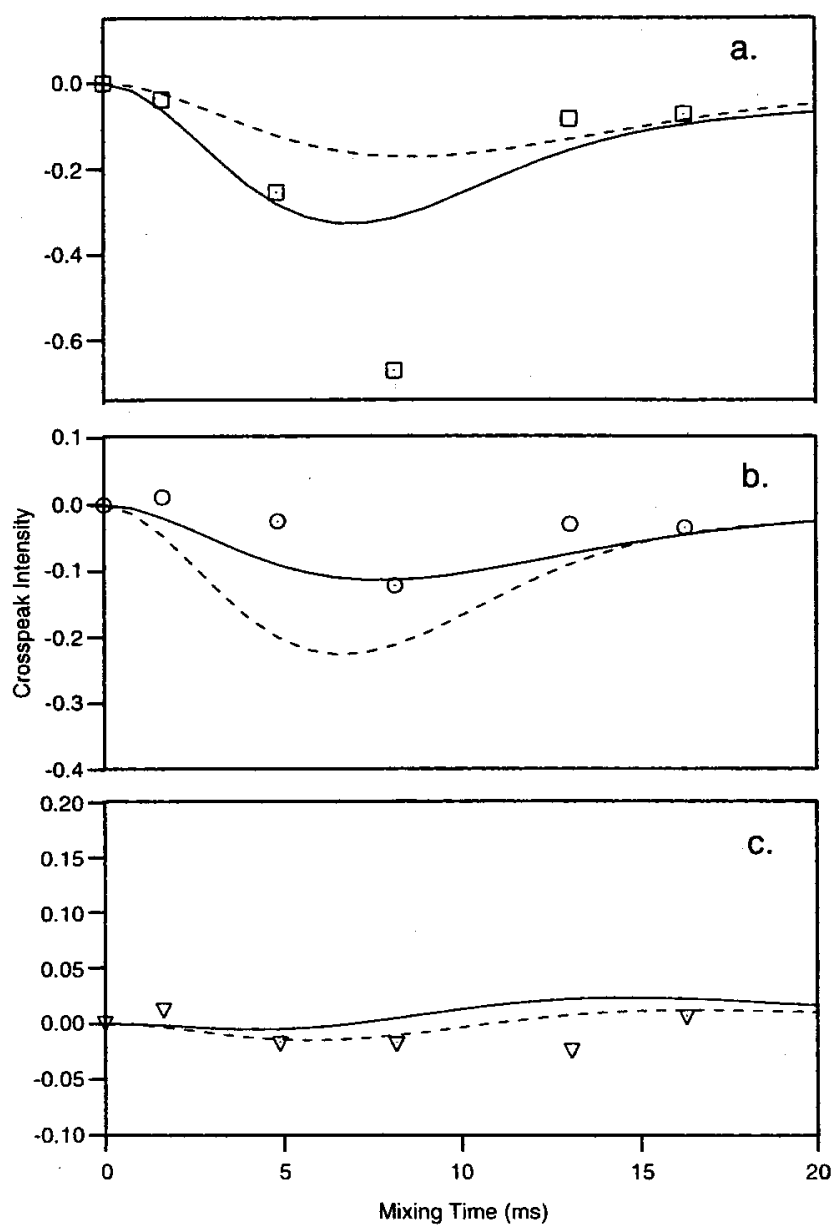

Figure 15. Comparison of experimental and simulated 2D DRAWS buildup curves for crystalline cytidine- $2,2^{\prime}, 5^{\prime}-{ }^{13} C_{3}$. Experimental crosspeak volumes were normalized to the auto-peak intensity in the first experiment. Simulations based on the crystal structure (essentially ideal A-form) are represented by solid lines, while those based on ideal B-form are shown by dashed lines. The $2-2^{\prime}$ cross-peak is shown in (a), $2^{\prime}-5^{\prime}$ in (b), and $2-5^{\prime}$ in (c). Relaxation rates and other parameters are given in Tables 7.1 and 7.2.

Such results indicate that there are considerable advantages to limiting the size of the spin system. As shown in Tables 3 and 4 , distance measurements from the smaller spin systems are considerably more precise than those from the uniformly labeled sample. Spectra in Figure 2 indicate that the lines are narrower, improving the resolution. As Figure 15 indicates, such information can be sufficiently precise to yield useful local conformations and thus, much structural information. The trade off is then between the precision of the measurements and the number of measurements obtained by uniform versus selective labeling of nucleosides.

\section{Conclusions}

The results of these studies clearly show that two-dimensional solid state dipolar recoupling experiments can be used to measure distances in selectively and uniformly labeled biomolecules. The accuracy of these measurements is dependent on the dipolar recoupling technique used in the experiments, the spin system geometry, and the spin system size. Simulations of data obtained from two doubly isotopically labeled cytidine samples indicate that the accuracy and precision of distance measurements by these two-dimensional techniques is, as expected, similar to that obtained in one-dimensional experiments. We also conclude that even when $R_{2 \mathrm{dq}}$ is treated as an 
adjustable parameter in 1D and 2D DRAWS simulations of doubly labeled crystalline cytidines, internuclear distances can be measured to roughly $\pm 0.2 \AA$ for distances in the range $3.1-$ $3.5 \AA$ either by numerical simulation or by simulations using average Hamiltonian theory. In doubly labeled cytidines, the most successful 2D RFDR simulations were accomplished using numerical methods.

Experiments and simulations carried out on the two triply labeled spin systems cytidine- $2,1^{\prime}, 3^{\prime}-{ }^{13} C_{3}$ and cytidine- $2,2^{\prime}, 5^{\prime}-$ ${ }^{13} C_{3}$ indicate that all three distances can be measured (with the exception of the $\mathrm{C} 2-\mathrm{C}^{\prime}$ ' distance in 2D DRAWS experiments), although the interpretation of the cross-peak intensity evolution associated with the longest distance is seriously complicated by indirect coherence transfer pathways. This interference between coherence transfer pathways can be directly observed as a alternation in the sign of cross-peak intensities in 2D DRAWS spectra (also reported for 2D MELODRAMA ${ }^{6}$ ), but is not easily observed in the 2D RFDR results. Simulations (see Figure 10) show that the precision and accuracy of long distance measurement is significantly reduced by interference between direct and indirect coherence transfer paths. Nevertheless, the precision and accuracy of the $\mathrm{C} 2-\mathrm{C} 3^{\prime}$ and $\mathrm{C} 2-\mathrm{C} 2$ ' distance measurements in triply labeled cytidines is sufficiently high to distinguish between the sugar ring puckering modes characteristic of the localized nucleic acid structure.

Simulations of the data obtained with 2D DRAWS for multiply labeled molecules can be simplified by the use of the average Hamiltonian expression while retaining sufficient accuracy to determine these conformations. Data obtained with 2D RFDR can be simulated with an exact Hamiltonian to yield accurate distances, although long distance measurements remain less precise due to indirect coherence transfer, as mentioned above.

Two-dimensional NMR spectra of uniformly labeled cytidine yielded a large number of distance constraints in these experiments, which could be obtained through average Hamiltonian simulations of segments of the full spin system. In addition, statistical analyses yielded linear correlations between crosspeaks from 2D RFDR and 2D DRAWS spectra to internuclear distances in the range $0.90-0.98$. However, due to the low precision of such measurements, structural detail in cytidine, including the sugar ring conformation, could not be determined, in this case. A major factor preventing detailed analysis of sugar ring pucker from uniformly labeled cytidine data was the degree to which indirect coherence transfer pathways dominate the weaker cross-peaks at very short mixing times, obscuring the long distance information needed to clearly distinguish between sugar ring puckering modes.

Based on these results, solid state NMR dipolar recoupling techniques can provide useful distance measurements under a variety of physical conditions. Numerous distances were obtained from two-dimensional spectra of uniformly labeled cytidine, but the low precision of such data did not allow the local conformation to be determined completely, suggesting that selective or random labeling may also be useful in studies of nucleic acids and related systems.

Two-dimensional NMR pulse sequences based on a zero quantum Hamiltonian (i.e., RFDR) and a double quantum Hamiltonian (i.e., DRAWS) have advantages and disdavantages, discussed above. A fruitful approach might involve the use of both types of experiments, i.e., to assign the resonances in a two-dimensional spectrum and to quantify cross-peak intensities in terms of internuclear distances.
Acknowledgment. The authors acknowledge support from NIH grant RO1-GM47802-04.

\section{References and Notes}

(1) Raleigh, D. P.; Levitt, M. H.; Griffin, R. G. Chem. Phys. Lett. 1988 , 146, 71. Gan, Z.-H.; Grant, D. M. Mol. Phys. 1989, 67, 1419-1430.

(2) Gullion, T.; Vega, S. Chem. Phys. Lett. 1992, 194, 423-428. Bennett, A.; Ok, J. H.; Vega, S.; Griffin, R. G. J. Chem. Phys. 1993, 96 , 8624-8627. Sodrickson, D. K.; Levitt, M. H.; Vega, S.; Griffin, R. G. J. Chem. Phys. 1993, 98, 6742-6748.

(3) Fujiwara, T.; Ramamoorthy, A.; Nagayama, K.; Hioka, K.; Fujlio, T. Chem. Phys. Lett. 1993, 212, 81-84. Baldus, M.; Tomaselli, M.; Meier, B. H.; Ernst, R. R. Chem. Phys. Lett. 1994, 230, 329-336.

(4) Lee, Y. K.; Kurur, N. D.; Helme, M.; Johannessen, O. G.; Nielsen, N C.; Levitt, M. H. Chem. Phys. Lett. 1995, 242, 304-309.

(5) Tycko, R.; Dabbagh, G. Chem. Phys. Lett. 1990, 173, 461-465.

(6) Sun, B.-Q.; Costa, P. R.; Kocisko, D.; Lansbury, P. T., Jr.; Griffin, R. G. J. Chem. Phys. 1995, 102, 702-707.

(7) Gregory, D. M.; Mitchell, D.; Stringer, J. A.; Kiihne, S. R.; Shiels, J. C.; Callahan, J.; Mehta, M. A.; Drobny, G. P. Chem. Phys. Lett. 1995, $246,654-663$.

(8) Tycko, R. J. Biomol. NMR 1996, 8, 239-251.

(9) Fujiwara, T.; Sugase, K.; Kainoshu, M.; Ono, A.; Akutsu, J. J. Am. Chem. Soc. 1995, 117, 11351-11352.

(10) Kiihne, S. R.; Mehta, M. A.; Stringer, J. A.; Gregory, D. M.; Shiels, J. C.; Drobny, G. P. J. Phys. Chem. 1998, A102, 2274-2282.

(11) Mehta, M. A.; Gregory, D. M.; Kiihne, S. R.; Mitchell, D. J.; Hatcher, M. E.; Shiels, J. C.; Drobny, G. P. Solid State NMR 1996, 7, $211-$ 228.

(12) Heller, J.; Larsen, R.; Ernst, M.; Kolbert, A. C.; Baldwin, M.; Prusiner, S. B.; Wemmer, D. E.; Pines, A. Chem. Phys. Lett. 1996, 251, 223-229.

(13) Long, J. R.; Dindot, J.; Zebroski, H.; Kiihne, S. R.; Clark, R. H.; Campbell, A. A.; Stayton, P. S.; Drobny, G. P. Proc. Natl. Acad. Sci. U.S.A 1998, 95, 12083-12087.

(14) Kline, P. C.; Serianni, A. S. J. Am. Chem. Soc. 1990, 112, 73737381

(15) Barker, R.; Fletcher, H. G. J. Org. Chem. 1961, 26, 4605-4609.

(16) Recondo, E. F.; Rinderknecht, G. L. Helv. Chim. Acta 1959, 42, $1171-1173$

(17) Codington, J. F.; Fox, J. J. Cytidine Sulfate; Codington, J. F.; Fox, J. J., Ed.; Academic Press: New York, 1963; Vol. 2, pp 113-116.

(18) Vorbrüggen, H.; Höfle, G. Chem. Ber. 1981, 114, 1256-1268.

(19) Batey, R. T.; Inada, M.; Kujawinski, E.; Puglisi, J. D.; Williamson, J. R. Nucl. Acids Res. 1992, 20, 4515-4523.

(20) Zimmer, D. P.; Crothers, D. M. Proc. Natl. Acad. Sci. U.S.A. 1995, 92, 3091-3095.

(21) Furberg, S.; Petersen, C. S.; Rømming, C. Acta Crystallogr. 1965 , $18,313-320$.

(22) Raleigh, D. P.; Olejniczak, E. T.; Griffin, R. G. J. Chem. Phys 1988, 89, 1333-1350.

(23) States, D. J.; Haberkorn, R. A.; Ruben, J. J. Magn. Reson. 1982 , $48,286-292$

(24) McKay 1990

(25) Herzfeld, J.; Berger, A. E. J. Chem. Phys. 1980, 73, 6021-6030.

(26) Gregory, D. M.; Mehta, M. A.; Shiels, J. C.; Drobny, G. P. J. Chem. Phys. 1997, 107, 28-42.

(27) McDowell, C. A.; Naito, A.; Sastry, D. L.; Takegoshi, K. J. Magn. Reson. 1988, 78, 498-510.

(28) Bevington, P. R.; Robinson, D. K. Data Reduction and Error Analysis for the Physical Sciences, 2nd ed.; McGraw-Hill: Boston, 1992.

(29) Santos, R. A.; Tang, P.; Harbison, G. S. Biochemistry 1989, 28, 9372-9378

(30) Geen, H.; Levitt, M. H.; Bodenhausen, G. Chem. Phys. Lett. 1992 200, 350-356.

(31) The MathWorks, I. Matlab, 5.0 ed.; The MathWorks, Ed.; PrenticeHall: Englewood Cliffs, NJ, 1996.

(32) Baldus, M.; Iuliucci, R. J.; Meier, B. H. J. Am. Chem. Soc. 1997, $119,1121-1124$

(33) Mortimer, R. G. Mathematics for Physical Chemistry; Mcmillan Publishing Co., Inc.: New York, 1981.

(34) Taylor, J. R. An Introduction to Error Analysis, 2nd ed.; University Science Books: Sausalito, CA, 1997.

(35) Wuthrich, K. NMR of Proteins and Nucleic Acids; John Wiley \& Sons: New York, 1986.

(36) Bax, A.; Lerner, L. J. Magn. Reson. 1988, 79, 429. 